\title{
Charge Transport in Disordered Graphene-Based Low Dimensional Materials
}

\author{
Alessandro Cresti ${ }^{1,2}$, Norbert Nemec ${ }^{3}$, Blanca Biel ${ }^{1,2}$, Gabriel Niebler ${ }^{4,5}$, François Triozon ${ }^{1}$, Gianaurelio \\ Cuniberti ${ }^{4}$, and Stephan Roche ${ }^{2}(\bowtie)$ \\ ${ }^{1}$ CEA, LETI, MINATEC, F38054 Grenoble, France \\ ${ }^{2}$ CEA, Institute for Nanoscience and Cryogenics, INAC/SPSMS/GT, 17 rue des Martyrs, 38054 Grenoble Cedex 9, France \\ ${ }^{3}$ Theory of Condensed Matter Group, Cavendish Laboratory, University of Cambridge, Trinity Lane, Cambridge CB2 17N, UK \\ ${ }^{4}$ Institute for Materials Science, TU Dresden, D-01062 Dresden, Germany \\ ${ }^{5}$ Department of Condensed Matter Physics, Faculty of Mathematics and Physics, Charles University, Ke Karlovu 5, 12116 Prague 2, \\ Czech Republic
}

Received: 11 August 2008/ Revised: 22 September 2008/Accepted: 23 September 2008

CTsinghua Press and Springer-Verlag 2008. This article is published with open access at Springerlink.com

\begin{abstract}
Two-dimensional graphene, carbon nanotubes, and graphene nanoribbons represent a novel class of low dimensional materials that could serve as building blocks for future carbon-based nanoelectronics. Although these systems share a similar underlying electronic structure, whose exact details depend on confinement effects, crucial differences emerge when disorder comes into play. In this review, we consider the transport properties of these materials, with particular emphasis on the case of graphene nanoribbons. After summarizing the electronic and transport properties of defect-free systems, we focus on the effects of a model disorder potential (Anderson-type), and illustrate how transport properties are sensitive to the underlying symmetry. We provide analytical expressions for the elastic mean free path of carbon nanotubes and graphene nanoribbons, and discuss the onset of weak and strong localization regimes, which are genuinely dependent on the transport dimensionality. We also consider the effects of edge disorder and roughness for graphene nanoribbons in relation to their armchair or zigzag orientation.
\end{abstract}

\section{KEYWORDS}

Graphene, charge transport, carbon nanotubes

\section{Introduction}

Since the discovery of fullerenes $\left(\mathrm{C}_{60}\right)$, carbon-based materials have been the subject of intense research, which led to the discovery of carbon nanotubes [14] and the fabrication of individual one-atom thick graphene layers [5]. This opens unprecedented avenues for the investigation of quantum transport in low dimensional (1-D and 2-D) systems, as well as attracting the interest of industry, given the potential for innovative applications. Carbon nanotube science is now a mature field of research, whose theoretical foundations have been reviewed in many textbooks and articles (see Refs. [3, 4] and references

Address correspondence to stephan.roche@cea.fr 
therein). More recently, the possibility of singling out a graphene plane, either through an exfoliation process [5], or by means of an epitaxial growth mechanism [6], has allowed the whole mesoscopic physics community to revisit basic foundations of quantum transport in 2-D systems, such as quantum Hall effects or weak localization phenomena [7-15], in a material with remarkable electronic properties [4]. Additionally, routes for an alternative carbonbased nanoelectronics are being actively investigated ranging from device optimization to graphene integration at the wafer scale $[6,16-21]$. However, since truly two-dimensional graphene is a zero-gap semiconductor, its use as an active electronic device, such as a field-effect transistor, requires the reduction of its lateral size to benefit from induced quantum confinement effects. Energy band gap engineering has been demonstrated with both carbon nanotubes (CNTs) and graphene nanoribbons (GNRs) [18, 22, 23]. In the case of GNR-based field-effect transistors, this results in an increase of performance with downscaling the ribbon width from several tens of $\mathrm{nm}$ to $2 \mathrm{~nm}$ [17-21, 24]. In contrast to CNTs, whose integration in operating devices at a large scale remains a tremendously complicated challenge, GNRs present the potential to be fabricated and massively integrated in complex architectures, thanks to the use of conventional lithographic techniques.

In this work, we discuss the transport properties of carbon-based low dimensional materials by focusing on the effect of a case of disorder potential, namely the Anderson-type model [25]. This model has been widely employed in studying the scaling theory of localization, and through analytical and numerical results, it allows us to overview the behavior of the basic transport length scales (elastic mean free path and localization lengths) and associated transport regimes in systems with different symmetries and transport dimensionality. In the case of GNRs, the presence of the edges exposes the system to further sources of disorder. In order to account for this peculiarity, we also consider the effects of impurities and roughness at the edges of the GNRs on the transport properties.

Section 1 is devoted to an overview of the electronic structure and quantum transport properties of 2-D-graphene, quasi-1-D CNTs and GNRs in the absence of disorder. We start from a simple nearest neighbor $(\mathrm{N}-\mathrm{N})$ tight-binding model and highlight differences and similarities between these different carbon structures due to their different dimensions and lack or presence of edges, as these are the underlying factors that determine the specific response of their transport properties to disorder. We include a discussion about how the electronic structure of narrow GNRs is modified when performing ab initio calculations and how to mimic this behavior by means of a third nearest neighbor tight-binding model. In the case of nanoribbons, we also elaborate on the dependence of the spatial symmetry of the system on the armchair or zigzag edge orientation and the number of carbon atoms in the primitive cell since, in certain cases, this has a deep impact on the transport features. At the end of the section, two interesting examples of transport in clean nanoribbons are also analyzed. The first one is the so called "pseudodiffusive" transport regime, which presents curious and intriguing analogies with the diffusive transport regime of disordered metals. The second one explores the effects of high magnetic fields on the energy bands, the transport properties, and the spatial distribution of electrical currents flowing through the ribbons.

In Section 2, we consider the effect of disorder on the density of states and the transport properties of the CNTs and GNRs. We focus on the analysis of typical length scales such as the mean free path and the localization length, taking an Andersontype disorder as a reference model. In spite of its simplicity, this type of disorder allows us to point out the differences between 2-D graphene and other graphene-derived materials. More realistic types of disorder would lead to more complicated effects that might mask the essential dependence of the transport properties on the dimensionality and symmetries of the investigated systems. A subsection is also devoted to the edge disorder (roughness and Anderson-type) in GNRs. The effect of this kind of disorder on the transport properties depends very critically on the armchair or zigzag edge orientation of the ribbons. In the literature, several different models of edge disorder have been considered and investigated 
with not always unanimous conclusions. In the last part of the section, we discuss the charge mobility in graphene-based systems subjected to different types of disorder, since this quantity is key to assess the performance of different devices.

Section 3 concludes the review by stressing the most relevant features.

\section{Electronic properties and transport in defect-free systems}

In most cases, the energetics of graphene-based materials can be conveniently described as a first approach by a $\pi$-effective electron model [4]. Indeed, for the honeycomb geometry, $\sigma$ bonds are formed by three out of four valence electrons ( $\mathrm{sp}^{2}$ hybridization), whereas the remaining single electron per carbon atom occupies a $2 p_{z}$ orbital whose hybridization with first neighbors generates $\pi$ and $\pi^{*}$ bands. A restriction of the Hamiltonian to a single $\mathrm{p}_{z}$ orbital per carbon atom thus provides an effective energetic model that well describes the electronic properties close to the charge neutrality point (CNP). In the case of GNRs, we assume that the $\sigma$ bonds at the edges are saturated with $\mathrm{H}$ atoms, thus eliminating dangling bonds.

\subsection{2-D Graphene band structure}

CNP locates the Fermi level for 2-D undoped graphene and metallic nanotubes and graphene ribbons, and the mid-gap of semiconducting CNTs and GNRs. Defining $\gamma_{0}$ as the integral overlap between $p_{z}$ orbitals, the electronic properties of 2-D graphene are straightforwardly derived by diagonalizing a $2 \times 2$ matrix as

$$
H(k)=\left(\begin{array}{cc}
0 & f^{*}(k) \\
f^{*}(k) & 0
\end{array}\right)
$$

with $f(\boldsymbol{k})=\gamma_{0} \sum_{m} \mathrm{e}^{\mathrm{i} k \boldsymbol{e}_{m}}\left(\boldsymbol{e}_{m}\right.$ are the vectors that connect $A$-type sites to the three nearest neighbor $B$-type sites, see Fig. 1 (a)) and $\gamma_{0} \approx-2.7 \mathrm{eV}$. It is readily shown that

$E_{ \pm}\left(k_{x}, k_{y}\right)= \pm \gamma_{0}\left[3+4 \cos \left(\frac{\sqrt{3} k_{x} a}{2}\right) \cos \left(\frac{k_{y} a}{2}\right)+2 \cos \left(k_{y} a\right)\right]^{1 / 2}$

(see Fig. 1(b)).

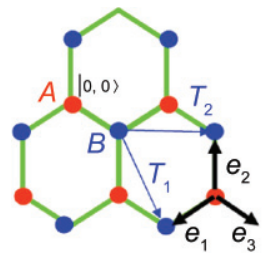

(a)

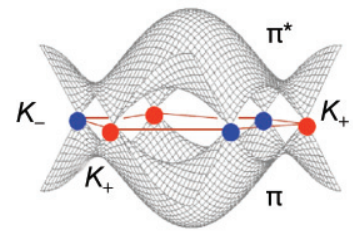

(b)

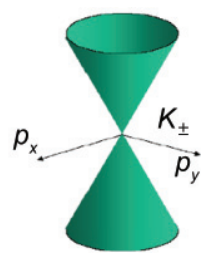

(c)
Figure 1 Honeycomb lattice, graphene $\pi$ and $\pi^{*}$ bands, and dispersion relation close to the $K_{ \pm}$points of the first Brillouin zone

The underlying symmetry and sublattice degeneracy of the honeycomb lattice can be unveiled from a different starting point. In a tight-binding representation, one can separate the two sublattices as

$$
H=\gamma_{0} \sum_{R \in \text { B.L. }} \sum_{m=1}^{3} B_{R+e_{m}}^{\dagger} A_{R}+\text { h.c. }
$$

by introducing creation $\left(A^{\dagger}, B^{\dagger}\right)$ and annihilation $(A, B)$ operators for electrons on site $A$ or $B$, respectively. To diagonalize the Hamiltonian, one applies the Bloch theorem to the Bravais lattice, which yields

$A_{R}^{\dagger}=\frac{1}{\sqrt{N_{m}}} \sum_{k \in 1 B Z} \mathrm{e}^{-\mathrm{i} k \cdot R} A_{k}^{\dagger} \quad B_{R+e_{m}}^{\dagger}=\frac{1}{\sqrt{N_{m}}} \sum_{k \in 1 B Z} \mathrm{e}^{-\mathrm{i} k \cdot\left(R+e_{m}\right)} B_{k}^{\dagger}$

with $N_{m}$ the number of unit cells. By means of this transformation,

$$
H=\sum_{k \in 1 B Z} h_{k} B_{k}^{\dagger} A_{k}
$$

with

$$
h_{k}=\gamma_{0} \sum_{m=1}^{3} \mathrm{e}^{-\mathrm{i} k \cdot e_{m}}=\left|h_{k}\right| \mathrm{e}^{\mathrm{i} \theta k}
$$

In the sublattice basis, at a given crystal momentum, this also is expressed as

$$
H=\sum_{k \in 1 B Z}\left(A_{k}^{\dagger}, B_{k}^{\dagger}\right)\left(\begin{array}{cc}
0 & h_{k} \\
h_{k} & 0
\end{array}\right)\left(\begin{array}{c}
A_{k} \\
B_{k}
\end{array}\right)
$$

Now, by performing a linearization close to $K$ points, i.e., $k=K_{ \pm}+\boldsymbol{p} / \hbar$ at first order in $p=-\mathrm{i} \hbar \nabla$, one finds

$$
\boldsymbol{H}_{K_{+}}=v_{F}\left(\begin{array}{cc}
0 & p_{x}+\mathbf{i} p_{y} \\
p_{x}-\mathbf{i} p_{y} & 0
\end{array}\right)
$$

where $v_{\mathrm{F}}=\sqrt{3} \alpha\left|\gamma_{0}\right| / 2 \hbar$ is the Fermi velocity. Therefore, the effective Hamiltonian takes the form of a Dirac Hamiltonian for massless particles

$$
H_{K_{+}}(p)=v_{\mathrm{F}} \boldsymbol{\sigma} \cdot p \quad \boldsymbol{H}_{K_{-}}(p)=-v_{\mathrm{F}} \boldsymbol{\sigma} \cdot \boldsymbol{p}
$$

with $\boldsymbol{\sigma}_{x, y, z}$ being the Pauli matrices. Finally, by identifying each of the $\boldsymbol{K}$-points with the index, these 
Hamiltonians can be rotated into a diagonal form with the unitary operator $\left(\theta=\arctan \left(p_{y} / p_{x}\right)\right)$

so that

$$
U_{\alpha}(p)=\frac{1}{\sqrt{2}}\left(\begin{array}{cc}
1 & 1 \\
-\alpha \mathrm{e}^{-\mathrm{i} \alpha \theta} & \alpha \mathrm{e}^{-\mathrm{i} \alpha \theta}
\end{array}\right)
$$

$$
\boldsymbol{U}_{\alpha}^{\dagger}(\boldsymbol{p}) \boldsymbol{H}_{\alpha}(\boldsymbol{p}) \boldsymbol{U}_{\alpha}^{\dagger}(\boldsymbol{p})=\alpha v_{F}\left(\begin{array}{cc}
-|p| & 0 \\
0 & |p|
\end{array}\right)=-\alpha v_{F}|\boldsymbol{p}| \boldsymbol{\sigma}_{z}
$$

The dispersion relation describes a cone-type structure in reciprocal space (see Fig. 1(c)), whereas the corresponding eigenvectors define a spinor (pseudospin) [4]

$$
\left|\boldsymbol{\Psi}_{p}^{+}\right\rangle=\frac{1}{\sqrt{2}}\left(\begin{array}{l}
\Psi_{p}^{+}(A) \\
\Psi_{p}^{+}(B)
\end{array}\right)=\frac{1}{\sqrt{2}}\left(\begin{array}{c}
\mathrm{se}^{\mathrm{i} \theta / 2} \\
\mathrm{e}^{-\mathrm{i} \theta / 2}
\end{array}\right)
$$

The spinor is also an eigenstate of the helicity operator $\hat{\varepsilon}=(1 / 2) \boldsymbol{\sigma} \cdot \boldsymbol{p} /|\boldsymbol{p}|$, such that $\hat{\varepsilon}\left|\boldsymbol{\Psi}_{p}^{+}(s= \pm 1)\right\rangle= \pm(1 / 2)\left|\boldsymbol{\Psi}_{p}^{+}(s)\right\rangle$. Thus, eigenstates have a well-defined helicity (good quantum number). Note, however, that the above derivation is valid in first order in $p$, whereas trigonal warping, which appears at second order [3], will bring substantial modifications of band structure with consequences on transport properties.

The electronic properties of the 2-D graphene can thus be described by an effective massless Dirac fermion model in the vicinity of the CNP, with linear dispersion and electron-hole symmetry. These properties derived close to the $K$ point are also valid for 1-D systems such as metallic nanotubes and wide armchair nanoribbons. However, other symmetries result in semiconducting systems with varying gaps. Semiconducting nanotubes and ribbons with increasing diameter (or width) show a linear downscaling of their associated energy gaps. By using proper boundary conditions, the electronic band structure of both types of systems can be analytically derived.

In the presence of a uniform magnetic field $B$ threading the 2-D graphene sheet along the orthogonal $z$ direction, we make use of the minimal substitution in the Hamiltonian. If we choose the first Landau gauge, the vector potential is $A=(B y, 0,0)$ and the new momentum operator is

$$
\boldsymbol{\pi}=\left(\begin{array}{c}
\pi_{x} \\
\pi_{y}
\end{array}\right)=\left(\begin{array}{c}
p_{x}-\frac{e}{c} B y \\
p_{y}
\end{array}\right)
$$

and so

$$
\left[\pi_{x}, \pi_{y}\right]=\mathrm{i} \frac{\hbar^{2}}{l_{0}^{2}} \quad \text { with } \quad l_{0}=\mathrm{i} \sqrt{\frac{c \hbar}{e B}}
$$

Let us define the following operators

$$
\eta=\frac{1}{\sqrt{2}} \frac{l_{0}}{\hbar}\left(\pi_{x}+\mathrm{i} \pi_{y}\right) ; \eta^{\dagger}=\frac{1}{\sqrt{2}} \frac{l_{0}}{\hbar}\left(\pi_{x}-\mathrm{i} \pi_{y}\right)
$$

These can be identified as annihilation and creation operators, since $\left[\eta, \eta^{\dagger}\right]=1$. The Hamiltonian around $\boldsymbol{K}_{+}$, is now

$$
\boldsymbol{H}_{k_{+}}=\beta\left(\begin{array}{ll}
0 & \eta \\
\eta^{\dagger} & 0
\end{array}\right) \quad \text { with } \quad \beta=\sqrt{2} \frac{\hbar v_{F}}{l_{0}}
$$

Let us consider the square of the Hamiltonian

$$
\boldsymbol{H}_{\boldsymbol{k}_{+}}^{2}=\beta^{2}\left(\begin{array}{cc}
\eta^{\dagger} \eta & 0 \\
0 & \eta \eta^{\dagger}
\end{array}\right)=\beta^{2}\left(\begin{array}{cc}
\eta^{\dagger} \eta & 0 \\
0 & \eta^{\dagger} \eta+1
\end{array}\right)
$$

We can identify $\eta^{\dagger} \eta$ as the number operator; therefore, the eigenvalues of $\boldsymbol{H}$ (given by the square root of the eigenvalues of $\boldsymbol{H}^{2}$ ) are [26]

$E_{n}= \pm \beta \sqrt{n} \approx 31.65 \sqrt{B} \sqrt{n} \quad$ with $n=0,1,2,3, \ldots$

where the eigenvalues $E_{n}$ are expressed in $\mathrm{meV}$ and the magnetic field $B$ in T. In contrast to ordinary 2-D electron gases, in graphene Landau levels are proportional to the square root of the magnetic field and to the square root of the integer number $n$. Moreover, a doubly degenerate zero energy level is present.

\subsection{Graphene nanoribbon band structure: Nearest neighbor tight-binding model}

Graphene nanonoribbons are strips of graphene that can be obtained by cutting a graphene sheet along a certain direction. Depending on this direction, the edges of the ribbon can be armchair-like (Fig. 2(a)) or zigzag-like (Fig. 3 (a)). In the following, we will refer to an armchair ribbon composed of $N$ dimer lines as $N$-aGNR, and to a zigzag ribbon composed of $N$ zigzag lines as $N$-zGNR.

The band structures of ideal GNRs with width below $\approx 100 \mathrm{~nm}$ and well defined edge symmetries (zigzag or armchair types) are dominated by confinement effects and van Hove singularities [2735], similarly to CNTs. As for 2-D graphene and CNTs, a nearest neighbor tight-binding model is found to describe low energy properties with a degree of approximation high enough for many 
applications. This approach has been widely employed for studying transport properties in pure or defected GNRs [27-29, 31, 33, 35-38].

\subsubsection{Armchair nanoribbons}

An armchair GNR unit cell contains $N$ A-type atoms and $N B$-type atoms, see Fig. 2(a). Thanks to the bipartite lattice of graphene, the total wavefunction of the system can be constructed by a linear combination of $A$-type $\Psi_{A}$ and B-type $\Psi_{B}$ sublattice wavefunctions. By applying Dirichlet boundary conditions for the wavefunctions at both edges of the ribbon $\left(q_{y}=2 p \pi /(\sqrt{3} a(N+1)), p=1,2, \ldots, N\right)$, one obtains [35]

$$
\begin{aligned}
(1 / N)|\Psi\rangle= & c_{A} \sum_{j=1}^{N} \sum_{x A_{j}} \mathrm{e}^{\mathrm{i} k x A_{j}} \sin \left(\sqrt{3} q_{y} a j / 2\right)\left|A_{j}\right\rangle+ \\
& c_{B} \sum_{j=1}^{N} \sum_{x B_{j}} \mathrm{e}^{\mathrm{i} k x B_{j}} \sin \left(\sqrt{3} q_{y} a j / 2\right)\left|B_{j}\right\rangle
\end{aligned}
$$

with $N=\sqrt{2 / N_{x}(N+1)}$ being a normalization factor. Then, by rewriting the Schrödinger equation as a 2 $\times 2$ matrix, eigenvalues and eigenvectors are readily found:

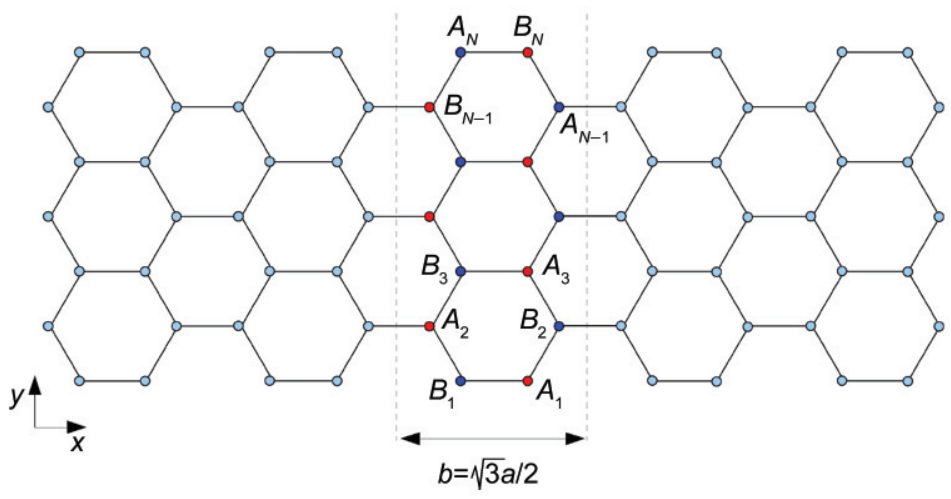

(a)

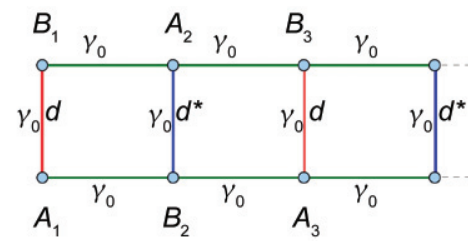

(b)

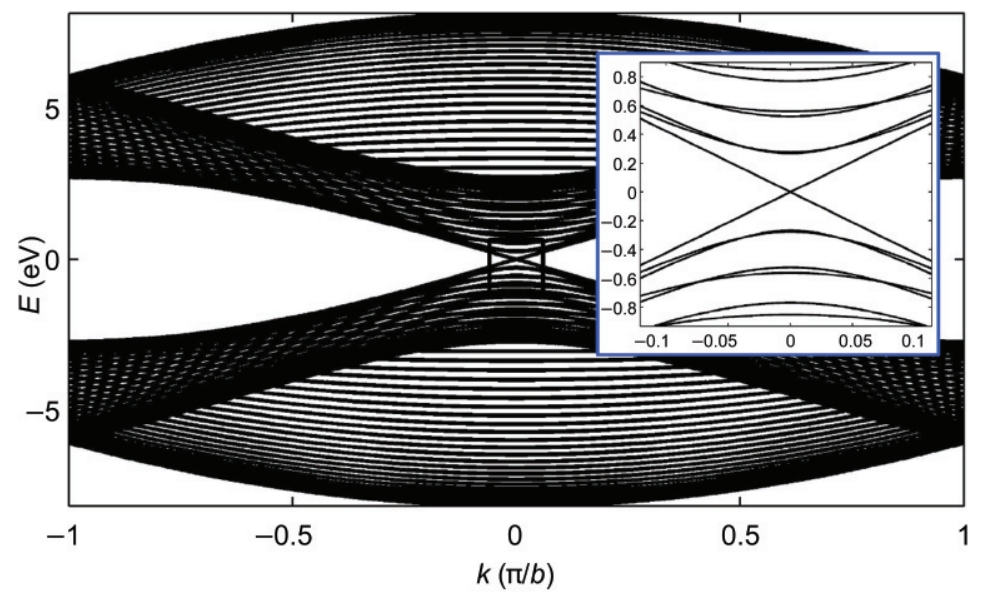

(c)

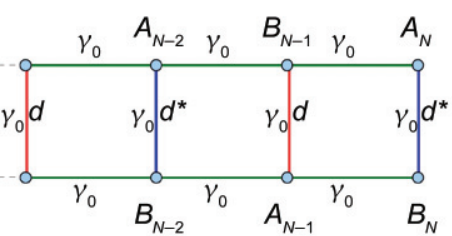

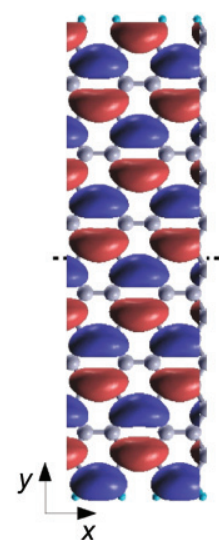

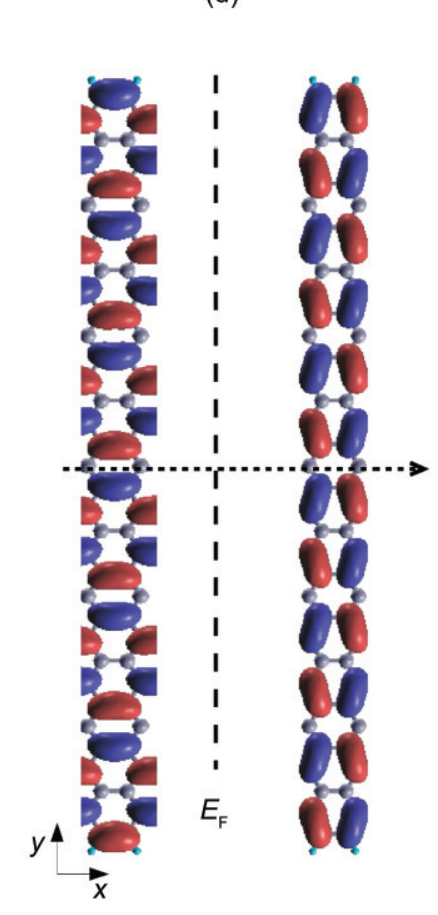

(e)

Figure 2 (a) Structure and primitive cell of an armchair ribbon composed of $N$ dimer lines; (b) equivalent coupled chains with $N$ orbitals each; (c) energy bands for a metallic ribbon with $N=53$; (d) 2-D projection (at the $\Gamma$ point) of the two wavefunctions just below and above the Fermi level for a 20-aGNR; (e) the same for a 35-aGNR. Blue and red colors correspond to opposite signs of the wavefunction

\section{(1)}




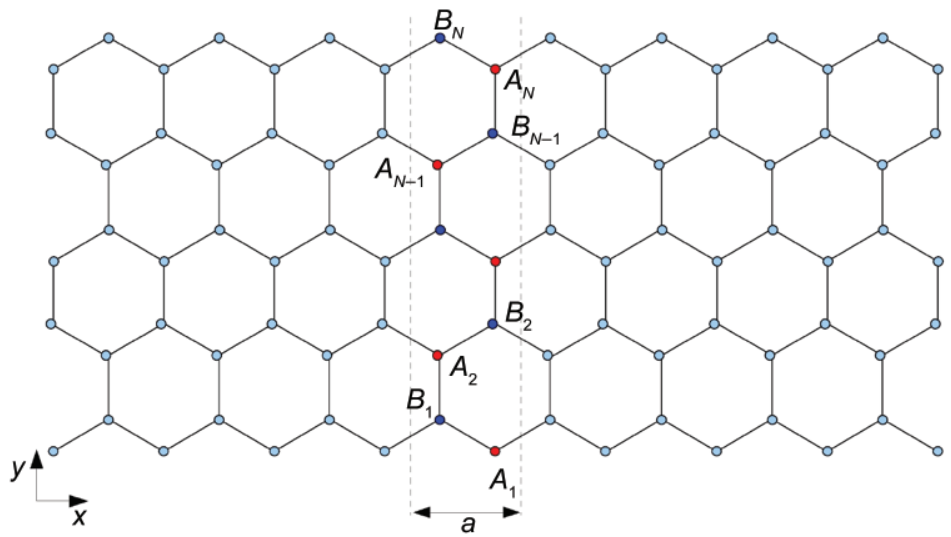

(a)

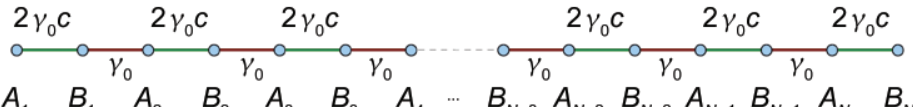

(b)

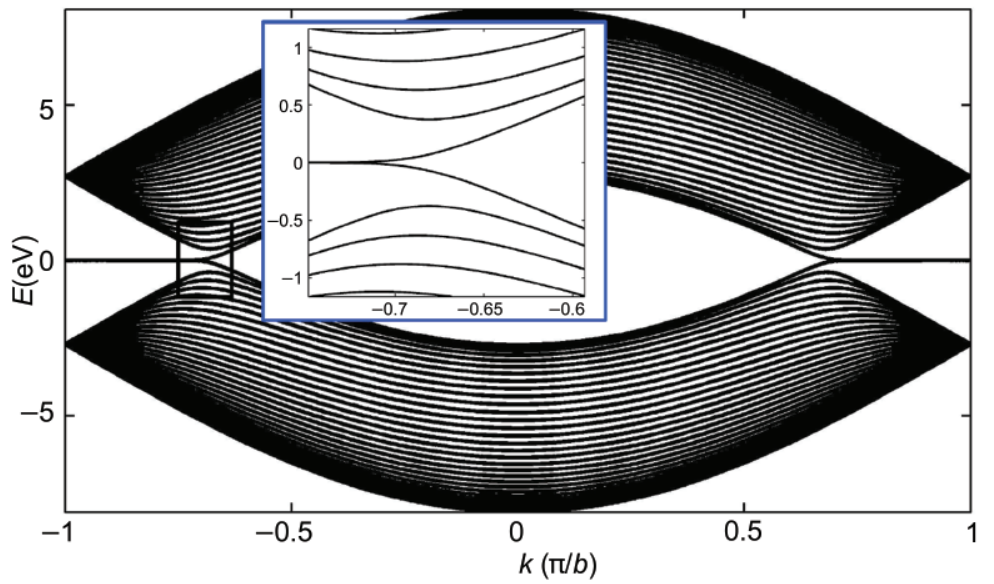

(c)

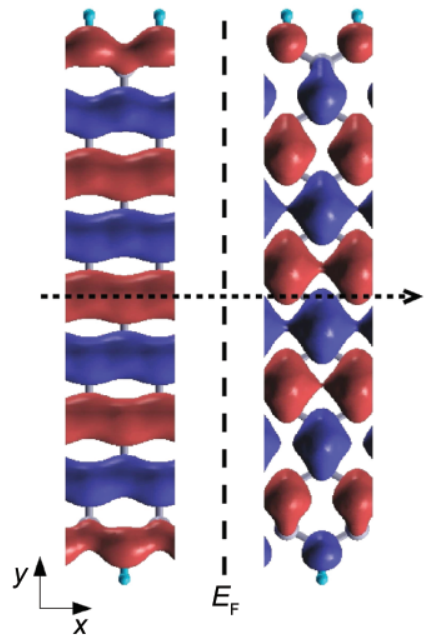

(d)

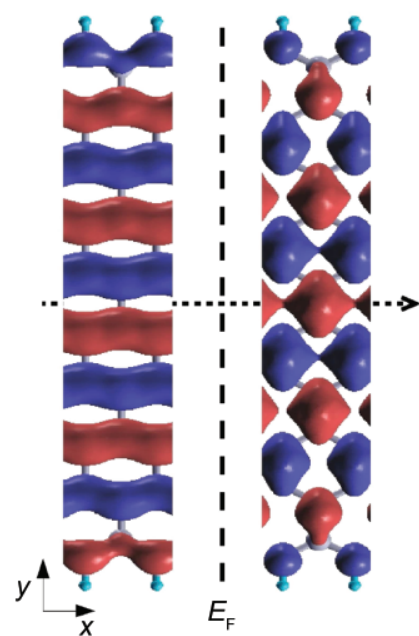

(e)

Figure 3 (a) Structure and primitive cell of a zigzag ribbon composed of $N$ carbon lines; (b) equivalent finite chain with $2 N$ orbitals; (c) energy bands for $N=32$; (d) 2-D projection (at the $\Gamma$ point) of the two wavefunctions just below and above the Fermi level for a 9-zGNR; (e) the same for the 10-zGNR. Blue and red colors correspond to opposite signs of the wavefunction

$$
\begin{aligned}
E\left(k, q_{y}\right) & = \pm\left|\gamma_{0}\left(2 \mathrm{e}^{\mathrm{i} k a / 2} \cos \left(\sqrt{3} q_{y} a / 2\right)+\mathrm{e}^{-\mathrm{i} k a}\right)\right| \\
& = \pm\left|\Gamma\left(k, q_{y}\right)\right|
\end{aligned}
$$

and

$$
|\Psi\rangle=1 / \sqrt{2}\left(\Psi_{A} \pm \sqrt{\Gamma^{*}\left(k, q_{y}\right) / \Gamma\left(k, q_{y}\right)} \Psi_{B}\right)
$$

By analyzing $\Gamma\left(k, q_{y}\right)$, we can see that the ribbon is metallic if $N=3 m+2$, where $m$ is an integer number, and semiconducting in the other cases. In particular, we can evaluate the energy gap $\Delta_{N}$ as a function of the $N$ dimer chains:

$$
\left\{\begin{array}{l}
\Delta_{3 m}=\left|\gamma_{0}\right|\left(4 \cos \frac{\pi m}{3 m+1}-2\right) \\
\Delta_{3 m+1}=\left|\gamma_{0}\right|\left(2-4 \cos \frac{\pi(m+1)}{3 m+2}\right) \\
\Delta_{3 m+2}=0
\end{array}\right.
$$

with $\Delta_{3 m}>\Delta_{3 m+1}>\Delta_{3 m+2}=0$. However, as reported in Section 1.3, ab initio calculations do not predict the existence of metallic ribbons and a give a different gap size hierarchy.

Further insight into the electronic structure of 
GNRs can be gained by representing the Hamiltonian on the Bloch sums. If we order the basis as $A_{1}$, $B_{2}, A_{3}, \ldots, A_{N-1}, B_{N}$ and $B_{1}, A_{2}, B_{3}, \ldots, B_{N-1}, A_{N}$, the Hamiltonian can be split into four $N \times N$ blocks

$$
H(k)=\gamma_{0}\left(\begin{array}{cccccccc}
0 & 1 & 0 & \ldots & d & 0 & 0 & \ldots \\
1 & 0 & 1 & \ldots & 0 & d^{*} & 0 & \ldots \\
0 & 1 & 0 & \ldots & 0 & 0 & d & \ldots \\
\ldots & \ldots & \ldots & \ldots & \ldots & \ldots & \ldots & \ldots \\
d^{*} & 0 & 0 & \ldots & 0 & 1 & 0 & \ldots \\
0 & d & 0 & \ldots & 1 & 0 & 1 & \ldots \\
0 & 0 & d^{*} & \ldots & 0 & 1 & 0 & \ldots \\
\ldots & \ldots & \ldots & \ldots & \ldots & \ldots & \ldots & \ldots
\end{array}\right)
$$

where $d(k)=\mathrm{e}^{\mathrm{i} k b / 2}$. The diagonal blocks are tridiagonal matrices with 0 diagonal elements and $\gamma_{0}$ offdiagonal elements, the off-diagonal blocks are diagonal matrices with alternating $\gamma_{0} \exp ( \pm \mathrm{i} k b / 2)$ elements. Hamiltonian (Eq. (23)) is equivalent to the Hamiltonian of two coupled chains with $N$ orbitals, as indicated in Fig. 2(b). Within each chain, the nearest neighbor orbitals are coupled by the hopping parameter $\gamma_{0}$ and have vanishing onsite energies. The interchain hopping is given by the parameters $\gamma_{0} \exp ( \pm \mathrm{i} k b / 2)$. By diagonalizing (Eq. (23)), the energy band structure is obtained. As an example, the energy bands of a metallic armchair ribbon with $N=53$ are reported in Fig. 2(c). Note the typical Diraclike linear dispersion around $k=0$. The mapping of the six Dirac points of 2-D graphene into $k=0$ can be easily understood by projecting the graphene band structure onto the axis corresponding to the armchair orientation [39]. At $k=0$, the Hamiltonian (Eq. (23)) is real and our coupled chains turn into a ladder with two legs and $N$ rungs and all hopping parameters equal to $\gamma_{0}$. The eigenvalues of such a system are analytically known $[40,41]$ and give again the energy gap $\Delta_{N}$ reported in Eq. (22).

The N-N tight-binding band structure of armchair nanoribbons with $N=3 m, 3 m+1$ and $3 m+2$ is summarized in Fig. 4 for $N=9,10$, and 11.

From the viewpoint of spatial symmetry, aGNRs, and GNRs in general, are very different from nanotubes. In fact, an ideal (perfect) CNT presents a well defined symmetry with respect to a large number of mirror planes containing the tube axis. As a consequence, the parity (even or odd) of the wavefunction has been demonstrated to have an impact on the transport properties of defected CNTbased systems, as scattering can only occur between eigenstates with the same parity [42-45]. On the contrary, wavefunctions in GNRs do not always present a well defined parity associated to mirror reflections with respect to the axis of the ribbon. The spatial symmetry depends on both the edge termination (zigzag or armchair) and the even or odd number of chains that comprise the ribbons. In the case of odd-indexed armchair ribbons (such as the 9-aGNR and 11-aGNR in Fig. 4), the system is invariant under mirror reflection with respect to a plane perpendicular to the ribbon and containing its axis. In the case of even-indexed armchair ribbons (such as the 10-aGNR in Fig. 4), the system is "asymmetric", in the sense that it is no longer invariant under the mirror reflection operation. Still, it is invariant under glide plane symmetry, which consists of the mirror reflection followed by a fractional translation along the ribbon axis. Figures $2(\mathrm{~d})$ and 2(e) show the 2-D-projection at the $\Gamma$ point of the two wavefunctions just below and above the Fermi level for perfect asymmetric (d) and symmetric (e) armchair ribbons (calculated with the SIESTA package [46]). As is clearly visible in Figs. 2(d) and 2(e), the existence of a mirror symmetry plane in ribbons leads to a well defined parity of the wavefunctions with respect to that mirror plane. The well defined (or not) parity of the wavefunctions does not affect the electronic transport properties of ideal unperturbed ribbons, but it may have a huge impact in the presence of defects. For example, the electronic transport properties of boron doped armchair ribbons have been shown, by means of ab initio calculations, to depend strongly on the symmetry of the ribbon, as $B$-induced potentials that preserve the parity of the wavefunctions do not affect the conductance of oddindexed ribbons at low energies. ${ }^{1}$ This suggests that scattering by certain defects might be suppressed, provided that the defects preserve the underlying symmetric geometry of the ribbon.

${ }^{1}$ Biel, B.; Blase, X.; Triozon, F.; Roche, S. submitted 


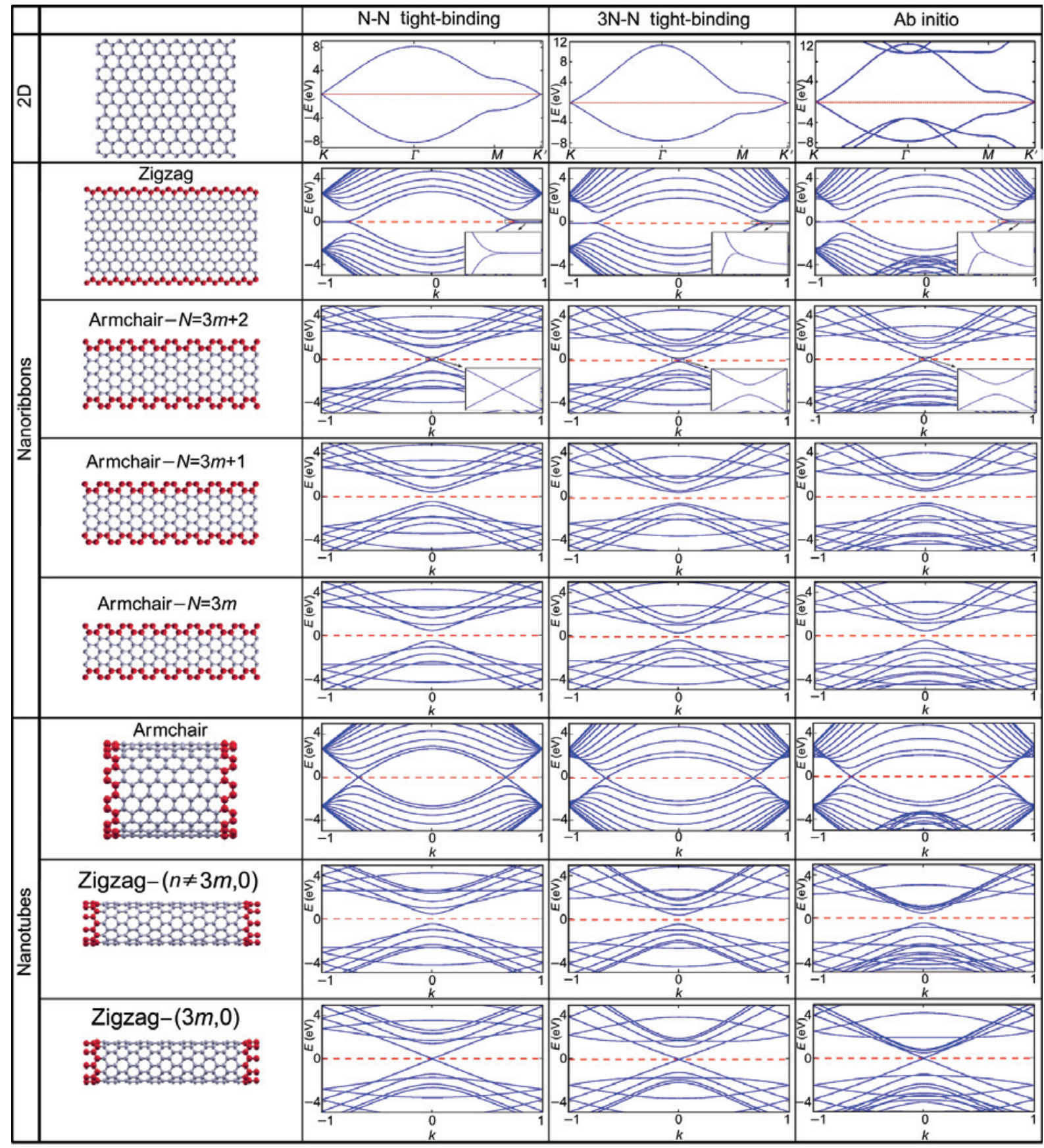

Figure 4 Band structures of 2-D graphene, $(9,0)$ and $(10,0)$ zigzag and $(10,10)$ armchair CNTs, and 10-zGNR, 9-, 10-, and 11-aGNRs obtained by the N-N tight-binding model, the third N-N tight-binding model and ab initio calculations. The energy bands of 2-D graphene are plotted along the $K-\Gamma-M-K^{\prime}$ direction. The energy bands of the GNRs and CNTs are plotted in the onedimensional first Brillouin zone. The wavevector $k$ is in units of $\pi$ divided by the lattice constant

\subsubsection{Zigzag nanoribbons}

For zGNRs, the handling of the boundary conditions is slightly more involved. A possible approach is solving the Dirac equation requiring that the wavefunction vanishes at the edges of the ribbon [32]. As evident from Fig. 3(a), the lower edge is entirely composed of $A$-type carbon atoms, while the upper edge is made up of B-type carbon atoms. Therefore, the boundary conditions can be imposed on the two sublattices separately. In particular, the wavefunction of the $A$-type sublattice is required to vanish on the upper edge and the wavefunction of the $B$-type 
sublattice is required to vanish on the lower edge. With these restrictions, the eigenfunctions of the Dirac equation can be separated into two groups. The first group includes states with wavenumber $k>1 / W$, where $W$ is the width of the ribbon. They are surface states and decay exponentially from the edges of the ribbon. The second group of eigenfunctions with $k<1$ / $W$ corresponds to confined states with nodes along the transverse section of the ribbon. To obtain the exact shape of these bands within the simple tightbinding approximation, a different approach can be used, viewing the GNR as a macromolecule with a basis of four carbon atoms [47].

In this case too, further information can be obtained by expressing the tight-binding Hamiltonian on the Bloch sums. It is easy to see that the $k$-dependent Hamiltonian is a tridiagonal $2 \mathrm{~N} \times 2 \mathrm{~N}$ matrix with alternating off-diagonal elements [48]

$$
H(k)=\gamma_{0}\left(\begin{array}{cccccc}
0 & 2 c(k) & 0 & \ldots & \ldots & 0 \\
2 c(k) & 0 & 1 & 0 & \ldots & 0 \\
0 & 1 & 0 & 2 c(k) & 0 & \ldots \\
\ldots & 0 & 2 c(k) & 0 & \ldots & \ldots \\
\ldots & \ldots & \ldots & \ldots & \ldots & 2 c(k) \\
0 & \ldots & \ldots & 0 & 2 c(k) & 0
\end{array}\right)
$$

where $c(k)=\cos (k a / 2)$ and the Bloch sums have been ordered as $A_{1}, B_{1}, A_{2}, \ldots, A_{N}, B_{N}$. This Hamiltonian is equivalent to the Hamiltonian of the $2 \mathrm{~N}$-orbital chain reported in Fig. 3(b). By diagonalizing matrix (Eq. (24)) numerically, the band structure is obtained for $N=32$, as shown in Fig. 3(c). The energy bands present some typical features: (1) the Dirac points of the 2-D graphene are mapped into $k= \pm 2 \pi /(3 a)$; (2) there are two partially flat degenerate bands with zero energy between the Dirac points and the border of the Brillouin zone, and the corresponding states are mainly located at the edges; (3) the bands are highly degenerate at the borders of the Brillouin zone; (4) at the Dirac points and close to the CNP the levels are equispaced. All these characteristics can be understood by simple considerations of the equivalent chain of Fig. 3(b). For example, at the edges of the first Brillouin zone we have $c(k= \pm \pi / a)=0$, thus the $2 \mathrm{~N}$-orbital chain splits into two isolated orbitals at its end and $N-1$ couples of dimers. This configuration generates two eigenstates with zero energy and located exactly on the edges of the ribbon, and (N-1)-fold degenerate eigenvalues $E= \pm \gamma_{0}$, as observed in point (3) above. If we consider a very large ribbon, our chain can be thought as semiinfinite. In this case, an analytical expression for the retarded Green's function projected on the first $\left(A_{1}\right)$ site can be obtained [48]

$G(E, k)=\frac{E^{2}-4 \gamma_{0}^{2} c^{2}+\gamma_{0}^{2} \pm \sqrt{\left(E^{2}-4 \gamma_{0}^{2} c^{2}+y_{0}^{2}\right)^{2}-4 \gamma_{0}^{2} E^{2}}}{4 \gamma_{0}^{2} E}$

where the sign in front of the square root is positive if $0<c(k) \leqslant 1 / 2$ and negative if $1 / 2<c(k) \leqslant 1$. In order to have a nonvanishing density of states (DoS), the discriminant under the square root must be negative, since the DoS is proportional to the imaginary part of the Green's function. Therefore, the four curves $E(k)= \pm \gamma_{0} \pm 2 \gamma_{0} \cos (k a / 2)$, along which the discriminant is vanishing, define the boundaries of the band structure and their intersection at $k= \pm 2 \pi /(3 a)$ identifies the folded Dirac points. As in the case of armchair nanoribbons, the mapping of the Dirac points can be predicted by the projection of the 2-D graphene bands onto the axis corresponding to the zigzag orientation. When $E \rightarrow 0$, the retarded Green's function has a continuum of poles for $0<c(k) \leqslant 1 / 2$, which correspond to the flat bands for $|k|>2 \pi /(3 a)$. The structure of the energy bands around the Dirac points and for finite width ribbons is obtained by considering that $c(k= \pm 2 \pi / 3 a)=1 / 2$, i.e., the hopping energies of the equivalent chain all become equal to $\gamma_{0}$. The eigenvalues of such a Hamiltonian are known [49] and, for large ribbons and around the CNP, can be approximated as

$E_{n}=\left|\gamma_{0}\right| \frac{\pi}{N+1 / 2}\left(n+\frac{1}{2}\right)$ with $n=0, \pm 1, \pm 2, \ldots$

As observed in point (4) above, this means that these levels are equispaced and the spacing is inversely proportional to the width of the ribbon itself.

The N-N tight-binding band structure of zigzag nanoribbons is summarized in Fig. 4 for $N=10$. As in the case of aGNRs, the even or odd number of chains that composes the ribbons determines the mirror or glide plane symmetry of zGNRs. Even-indexed zigzag ribbons (such as the 10-zGNR in Fig. 4) are invariant under mirror reflection with respect to a plane perpendicular to the ribbon and containing 
its axis. Odd-indexed zigzag ribbons are invariant under glide plane symmetry. The parity of the wavefunctions with respect to the spatial symmetry of the GNR is clearly visible in Figs. 3(d) and 3(e), where we show the 2-D-projection at the $\Gamma$ point of the two wavefunctions just below and above the Fermi level for perfect asymmetric (Fig. 3(d)) and symmetric (Fig. 3(e)) zigzag ribbons (calculated with the SIESTA package [46]). The spatial symmetry of zGNRs and the respective parity of the wavefunctions play an important role in determining the electronic transport properties in the presence of external fields [50-54]. For example, a valley-valve effect (blocking of the electrical current by a p-n junction) has been demonstrated theoretically in zigzag ribbons in the presence of an external potential [50]. This effect depends critically on the parity of the number of zigzag chains across the ribbons [51] and has been successfully explained by realizing that, in case of even-indexed zGNRs, the symmetry of the ribbons is preserved by the considered superimposed potential, thus forbidding potential-induced transitions between opposite parity eigenstates [53, 54]. A similar explanation has been found for the case of an applied bias in zigzag ribbons, when transitions between the valence and the conduction bands are only allowed for asymmetric ribbons [52], and the opening of a conductance gap in the vicinity of the Fermi level is expected for symmetric zigzag ribbons. This intriguing particularity has no analogue in nanotubes.

\subsubsection{Carbon nanotube band structure}

As for GNRs, the electronic properties of nanotubes are strongly modulated by small structural variations; in particular, their metallic or semiconducting character is determined by the diameter and helicity (chirality) of the carbon atoms in the tube. This dependence is easily understood starting from the energy dispersion relations of graphene around the Fermi level, by retaining only the $\pi-\pi^{*}$ bands. Rolling the graphene into a cylinder imposes periodic boundary conditions for the wavefunctions along the circumference of the tube and results in a quantization of the momentum component along this direction. For details of the derivation of the tight-binding graphene dispersion relations, and the subsequent zone-folding procedure to obtain the band-structure of nanotubes, we refer the reader to Ref. [4]. The bands obtained for armchair tubes with helicity $(N, N)$ are, for instance, given by

$$
E_{q}^{ \pm}(k)= \pm \gamma_{0} \sqrt{1 \pm 4 \cos \frac{k a}{2} \cos \frac{q \pi}{N}+4 \cos ^{2} \frac{k a}{2}}
$$

where $q(=1,2, \ldots, 2 N)$ specifies the discrete part of the wavevector perpendicular to the tube axis (i.e., the band index), $k$ is the continuous component that describes eigenstates in a given subband $(-\pi<k a<\pi)$ and $a=2.46 \AA$ is the graphene lattice constant.

The band structures for the armchair $(10,10)$ and the zigzag $(9,0)$ and $(10,0)$ nanotubes calculated within the N-N tight-binding model are shown in Fig. 4.

\section{3 $\mathrm{Ab}$ initio results}

In spite of the good description the most simplified $\mathrm{N}-\mathrm{N} \pi$-orbital tight-binding model provides for the electronic properties of 2-D-graphene and CNTs, some particular features regarding mainly the effect of edges in GNRs have only been unveiled by means of ab initio simulations [55-60] or more sophisticated tight-binding models [61-63]. The stability of edge states in zGNRs has been analyzed both with and without $\mathrm{H}$ atoms by means of ab initio simulations. The almost flat bands in the vicinity of the Fermi level, originated by edge states, become more dispersive, thus producing a peak in the conductance of the system, which jumps from one to three conduction channels in a region of $\sim 0.3 \mathrm{eV}$ below the $\mathrm{CNP}$ of the ideal ribbon. The dispersion at the edge state predicted by first-principles calculations has been attributed to the interaction with next-nearest neighbors, that decreases the energy eigenvalue of the edge state [62], though there are still some discrepancies with respect to experimental data [6466]. However, the metallic character of zigzag ribbons is preserved as long as the spin degree of freedom is not taken into account [57].

On the other hand, ab initio studies have demonstrated that there are no truly metallic armchair GNRs [58-60]. Even for those armchair ribbons predicted to be metallic by the N-N tight-binding model, a gap opens, thus modifying their character 
from metallic to semiconducting. The magnitude of the gap, however, decreases with increasing ribbon width, and for a ribbon of $\sim 5 \mathrm{~nm}$ with a metallic behavior predicted by the N-N tight-binding model, the ab initio estimated band gap is only $\sim 0.05 \mathrm{eV}$. The magnitude of the gaps of the semiconducting ribbons predicted by the N-N tight-binding model also differs from those estimated by means of first-principles calculations. There have been several explanations for the origin of these gaps, supported by more sophisticated tight-binding models that take into account edge distortion $[59,61]$, up to three nearest neighbor interactions [60], or both [63].

The ab initio band structure of 2-D graphene and different types of nanoribbons and nanotubes are shown in the last column of Fig. 4 . All the ab initio calculations were performed with the SIESTA package [46].

\subsection{Third nearest neighbor interaction tight- binding model}

\subsubsection{Changes in 2-D graphene band structure}

In Section 1.1, it was mentioned that the band structure of 2-D graphene can be calculated using the nearest neighbor tight-binding approximation [67]. This leads to Eq. (2), which is plotted in Fig. 1 (b) and Fig. 4. We will now see how this result is modified by taking into account higher orders of interaction and orbital overlap.

Figure 5(a) graphically shows the distances between first, second, and third nearest neighbors. These distances are given by $d_{1}=1.42 \AA, d_{2}=\sqrt{3} d_{1} \approx$ $1.73 d_{1}$ and $d_{3}=2 d_{1}$. From Fig. 5(a), we also see that every atom in the graphene lattice has three nearest neighbors, six second nearest neighbors and three third nearest neighbors. The small difference in length between the distances of second and third nearest neighbors $\left(d_{2}\right.$ and $\left.d_{3}\right)$ suggests the inclusion of both of these interactions. Figure 5(b) allows us to evaluate which interactions exist between the unit cell here labeled $(0,0)$ and its neighboring unit cells. We can therefore write the tight-binding Hamiltonian, including up-to-third nearest neighbor interaction:
$\boldsymbol{H}(\boldsymbol{k})=\boldsymbol{H}_{0,0}+\mathrm{e}^{\mathrm{i} k \cdot \boldsymbol{a}_{1}} \boldsymbol{H}_{1,0}+\mathrm{e}^{-\mathrm{i} k \cdot \boldsymbol{a}_{1}} \boldsymbol{H}_{-1,0}+\mathrm{e}^{\mathrm{i} k \cdot \boldsymbol{a}_{2}} \boldsymbol{H}_{0,1}+\mathrm{e}^{-\mathrm{i} k \cdot \boldsymbol{a}_{2}} \boldsymbol{H}_{0,-1}$

$+\mathrm{e}^{\mathrm{i} k \cdot\left(a_{1}+a_{2}\right)} \boldsymbol{H}_{1,1}+\mathrm{e}^{-\mathrm{i} k \cdot\left(a_{1}+a_{2}\right)} \boldsymbol{H}_{-1,-1}+\mathrm{e}^{\mathrm{i} k \cdot\left(a_{1}-a_{2}\right)} \boldsymbol{H}_{1,-1}+\mathrm{e}^{-\mathrm{i} \boldsymbol{k} \cdot\left(a_{1}-a_{2}\right)} \boldsymbol{H}_{-1,1}$

where $a_{1}=(\sqrt{3}, 3) a / 2$ and $a_{2}=(\sqrt{3},-3) a / 2$ are the translation vectors of the hexagonal lattice and

$\boldsymbol{H}_{0,0}=\left(\begin{array}{rr}\varepsilon_{0} & -\gamma_{0} \\ -\gamma_{0} & \varepsilon_{0}\end{array}\right), \boldsymbol{H}_{1,-1}=\left(\begin{array}{ll}-\gamma_{1} & -\gamma_{2} \\ -\gamma_{2} & -\gamma_{1}\end{array}\right), \boldsymbol{H}_{0,1}=\left(\begin{array}{cc}-\gamma_{1} & -\gamma_{0} \\ 0 & -\gamma_{1}\end{array}\right)$,

$\boldsymbol{H}_{1,1}=\left(\begin{array}{cc}0 & -\gamma_{2} \\ 0 & 0\end{array}\right), \boldsymbol{H}_{0,-1}=\left(\begin{array}{cc}-\gamma_{1} & 0 \\ -\gamma_{0} & -\gamma_{1}\end{array}\right), \boldsymbol{H}_{-1,-1}=\left(\begin{array}{cc}0 & 0 \\ -\gamma_{2} & 0\end{array}\right)$

with $\boldsymbol{H}_{i, j}=\boldsymbol{H}_{i, i}$ for $i \neq j$. First, second, and third nearest neighbor hopping constants are here called $\gamma_{0}, \gamma_{1}$ and $\gamma_{2}$, in accordance with Fig. 5(b). The onsite energy is indicated by $\varepsilon_{0}$. Analogously, we define $\sigma_{0}, \sigma_{1}$, and $\sigma_{2}$ to be the overlap matrix elements between first, second and third nearest neighbors, respectively, and thus obtain a formula for $S$ that looks just like Eq. (28), with every $H$ replaced by an $S$ and

$S_{0,0}=\left(\begin{array}{cc}1 & \sigma_{0} \\ \sigma_{0} & 1\end{array}\right), S_{1,-1}=\left(\begin{array}{cc}\sigma_{1} & \sigma_{2} \\ \sigma_{2} & \sigma_{1}\end{array}\right), S_{0,1}=\left(\begin{array}{cc}\sigma_{1} & \sigma_{0} \\ 0 & \sigma_{1}\end{array}\right)$,

$S_{1,1}=\left(\begin{array}{cc}0 & \sigma_{2} \\ 0 & 0\end{array}\right), S_{0,-1}=\left(\begin{array}{cc}\sigma_{1} & 0 \\ \sigma_{0} & \sigma_{1}\end{array}\right), S_{-1,-1}=\left(\begin{array}{cc}0 & 0 \\ \sigma_{2} & 0\end{array}\right)$

where of course $S_{i, j}=S_{i, i}$ for $i \neq j$. We now define

$$
\begin{aligned}
& g_{0}(\boldsymbol{k})=1+\mathrm{e}^{\mathrm{i} k \cdot a_{1}}+\mathrm{e}^{\mathrm{i} k \cdot a_{2}} \\
& g_{1}(\boldsymbol{k})=\mathrm{e}^{\mathrm{i} k \cdot a_{1}}+\mathrm{e}^{-\mathrm{i} k \cdot a_{1}}+\mathrm{e}^{\mathrm{i} k \cdot a_{2}}+\mathrm{e}^{-\mathrm{i} k \cdot a_{2}}+\mathrm{e}^{\mathrm{i} k \cdot\left(a_{1}-a_{2}\right)}+\mathrm{e}^{-\mathrm{i} k \cdot\left(a_{1}-a_{2}\right)} \\
& g_{2}(\boldsymbol{k})=\mathrm{e}^{\mathrm{i} k \cdot\left(a_{1}+a_{2}\right)}+\mathrm{e}^{\mathrm{i} k \cdot\left(a_{1}-a_{2}\right)}+\mathrm{e}^{-\mathrm{i} k \cdot\left(a_{1}-a_{2}\right)}
\end{aligned}
$$

which permits us to write the Hamiltonian $\boldsymbol{H}$ as

$$
\boldsymbol{H}=\left(\begin{array}{cc}
\varepsilon_{0}-\gamma_{1} g_{1}(\boldsymbol{k}) & -\gamma_{0} g_{0}(\boldsymbol{k})-\gamma_{2} g_{2}(\boldsymbol{k}) \\
-\gamma_{0} g_{0}^{*}(\boldsymbol{k})-\gamma_{2} g_{2}^{*}(\boldsymbol{k}) & \varepsilon_{0}-\gamma_{1} g_{1}(\boldsymbol{k})
\end{array}\right)
$$

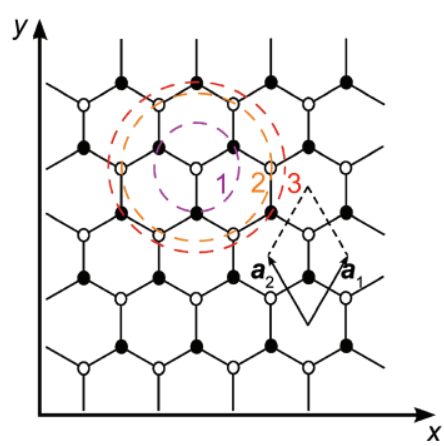

(a)

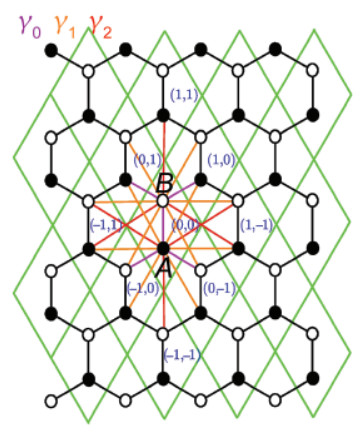

(b)
Figure 5 (a) An atom in the graphene lattice with its first, second, and third nearest neighbors, indicated by three dashed circles. Second nearest neighbors belong to the same sublattice of the considered atom, first and third nearest neighbors to the opposite sublattice type. (b) A unit cell of the graphene lattice and its adjacent unit cells. Boundaries between unit cells are marked in green, and are labeled in blue. Purple lines represent first, orange lines second, and red lines third nearest neighbor 
and the overlap matrix $S$ as

$$
S=\left(\begin{array}{cc}
1+\sigma_{1} g_{1}(\boldsymbol{k}) & \sigma_{0} g_{0}(\boldsymbol{k})+\sigma_{2} g_{2}(\boldsymbol{k}) \\
\sigma_{0} g_{0}^{*}(k)+\sigma_{2} g_{2}^{*}(\boldsymbol{k}) & 1+\sigma_{1} g_{1}(\boldsymbol{k})
\end{array}\right)
$$

Now all that is left to do is to solve the generalized characteristic equation,

$$
\begin{aligned}
& |\boldsymbol{H}-E \boldsymbol{S}|=\left|\begin{array}{ll}
H_{A A}-E S_{A A} & H_{A B}-E S_{A B} \\
H_{A B}^{*}-E S_{A B}^{*} & H_{A A}-E S_{A A}
\end{array}\right|=0 \\
\Rightarrow & \left(H_{A A}-E S_{A A}\right)^{2}-\left(H_{A B}-E S_{A B}\right)\left(H_{A B}^{*}-E S_{A B}^{*}\right)=0 \\
\Rightarrow & \overbrace{}^{2}(\overbrace{S_{A A}^{2}-\left|S_{A B}\right|^{2}}^{\varepsilon_{1}}+E(\overbrace{H_{A B} S_{A B}^{*}+H_{A B}^{*} S_{A B}}^{\varepsilon_{2}}-\overbrace{H_{A A} S_{A A}}^{\varepsilon_{3}})+ \\
& \overbrace{H_{A A}^{2}-\left|H_{A B}\right|^{2}}^{\varepsilon_{A}}=0
\end{aligned}
$$

where we introduce $\varepsilon_{1,2,3,4}$ as a shorthand notation. From comparison between Eqs. (32) and (33) we see that

$$
\begin{aligned}
& \varepsilon_{1}=\left(1+\sigma_{1} g_{1}(\boldsymbol{k})\right)^{2}-\left|\sigma_{0} g_{0}(\boldsymbol{k})+\sigma_{2} g_{2}(\boldsymbol{k})\right|^{2} \\
& \varepsilon_{2}=-\left[\left(\gamma_{0} g_{0}(\boldsymbol{k})+\gamma_{2} g_{2}(\boldsymbol{k})\right) \times\left(\sigma_{0} g_{0}^{*}(\boldsymbol{k})+\sigma_{2} g_{2}^{*}(\boldsymbol{k})\right)+c . c .\right] \\
& \varepsilon_{3}=\left(\varepsilon_{0}-\gamma_{1} g_{1}(\boldsymbol{k})\right) \times\left(1+\sigma_{1} g_{1}(\boldsymbol{k})\right) \\
& \varepsilon_{4}=\left(\varepsilon_{0}-\gamma_{1} g_{1}(\boldsymbol{k})\right)^{2}-\left|\gamma_{0} g_{0}(\boldsymbol{k})+\gamma_{2} g_{2}(\boldsymbol{k})\right|^{2}
\end{aligned}
$$

We are thus left with the following dispersion relation for 2-D graphene:

$$
E^{ \pm}=\frac{-\left(\varepsilon_{2}-2 \varepsilon_{3}\right) \pm \sqrt{\left(\varepsilon_{2}-2 \varepsilon_{3}\right)^{2}-4 \varepsilon_{1} \varepsilon_{4}}}{2 \varepsilon_{1}}
$$

The values for $\gamma_{0-2}$ and $\sigma_{0-2}$ have been calculated by Reich et al. by comparing and fitting band structures from ab initio and tight-binding calculations [68]. The third nearest neighbor tight-binding band structure with these parameters is plotted in Fig. 4. Recently, more sophisticated GW ( $G=$ Green's function, $W=$ screened Coulomb interaction) ab initio calculations [69] for bilayer and few-layer graphene provided $\sim 20 \%$ larger values of $\gamma_{0-2}$. This is in better agreement with some experimental measurements, in particular angle-resolved photoemission (ARPES), where the long range Coulomb interaction plays an important role and the band structure at high energies requires a more accurate description.

\subsubsection{Effects on the band structure of GNRs}

It has already been mentioned that the metallicity of armchair GNRs of widths $N=3 m+2(m \in N)$ is only found in the N-N tight-binding approximation, while ab initio calculations show all armchair GNRs to be semiconducting (cf. Section 1.2 and Section 1.3). This finding is reproduced by third nearest neighbor tight-binding calculations. The gap opening can be quantified by comparing $\mathrm{N}-\mathrm{N}$ and third nearest neighbor (3N-N) tight-binding results with each other (see Fig. 6). The relative opening or closing of the band gap decreases with the width as $1 / N$. Furthermore, it has been shown that edge deformations [34, 35] and strain $[70,71]$ significantly change the band gap of armchair GNRs, turning metallic ribbons into semiconducting ones, even when calculated with the N-N tight-binding model. The metallicity of certain armchair ribbons should therefore be considered an artifact of the N-N tight-binding approximation and not a robust feature of these ribbons.

In the case of zGNRs the 3N-N tight-binding model shows robust metallicity and a higher

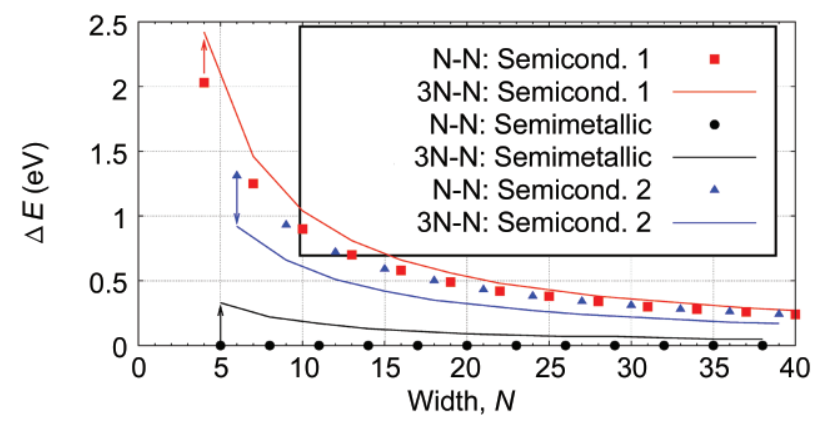

(a)

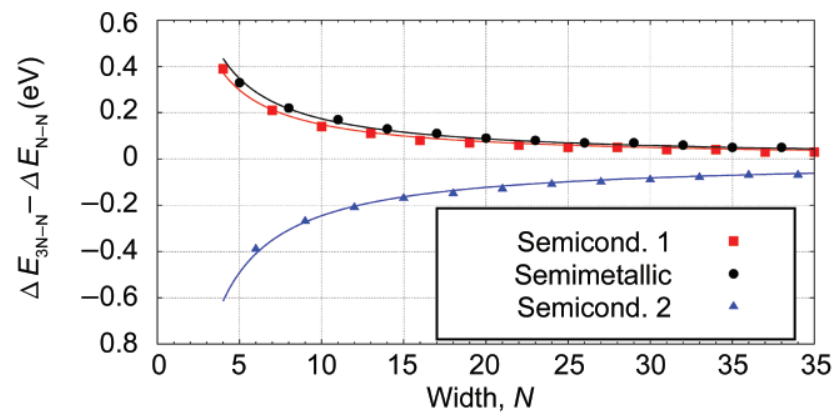

(b)

Figure 6 (a) Band gaps of armchair GNRs as a function of their width $\mathrm{N}$ in N-N and $3 \mathrm{~N}-\mathrm{N}$ tight-binding. Because of the three-fold periodicity in the band gap width, it is convenient to plot three different curves or sets of points corresponding to $\bmod (N, 3)=0,1,2$. The symbols show N-N results, and the lines the corresponding $3 \mathrm{~N}-\mathrm{N}$ results. (b) The gap opening/closing obtained from a comparison of $\mathrm{N}-\mathrm{N}$ and $3 \mathrm{~N}-\mathrm{N}$ tight-binding results. The symbols show the opening (or closing) of the band gap in armchair GNRs of the respective widths. The lines are fitted to $\alpha / N$, where $\alpha$ is the fitting parameter. This is analogous to the case of zigzag carbon nanotubes in which the gap decreases as $1 / d$, where $d$ is the diameter of the tube 
dispersion of the edge state than in the case of only nearest neighbor interaction (cf. Fig. 4). The full ab initio results show a band gap that opens due to magnetic ordering [59]. This feature is not reproduced by the tight-binding model employed here, but one can account for such spin-related effects by including a Hubbard term in the Hamiltonian [39, 72-74].

It can thus be said that the third nearest neighbor tight-binding method leads to significantly better results than the nearest neighbor method when compared to ab initio results, while keeping within the simplicity of the tight-binding model.

The 3N-N energy bands for 2-D graphene and different types of nanoribbons and nanotubes are summarized in the fourth column of Fig. 4.

\subsection{Transport in nanotubes and nanoribbons}

When neglecting disorder effects, a CNT of length $L$ between metallic contact reservoirs is a ballistic conductor with an $L$-independent conductance given by the energy-dependent number of available quantum channels $N_{\perp}(E)$. Each channel carries one conductance quantum $G_{0}=2 e^{2} / h$, thus $G(E)=2 e^{2} / h \times$ $N_{\perp}(E)$, including spin degeneracy. This only occurs in the case of perfect (reflection-less) or ohmic contacts between the CNT and metallic voltage probes [75, 76]. In this regime, the expected energy-dependent conductance spectrum is easily deduced from band structure calculations, by counting the number of channels at a given energy. For instance, armchair nanotubes present two quantum channels at the CNP, which result in $G\left(E_{\mathrm{F}}\right)=2 G_{0}$. At higher energies, the ballistic conductance increases as more channels become available to conduction.

In contrast, the eigenstates of defect-free armchair GNRs in the first plateau are constrained by the hardwall (Dirichlet) boundary conditions which require a node at the edges, thus excluding the cosine solutions [36]. As a result, a singly degenerate band is left in the first conductance plateau.

Within the linear regime near the CNP, a closed expression for the the number of channels is given by

$$
N_{\perp}(E)=2+4\left(\frac{d}{2 \hbar v_{\mathrm{F}}}\left|E-E_{\mathrm{F}}\right|\right)
$$

in the case of an armchair CNT. For CNTs of other chiralities and GNRs, similar expressions can be obtained.

1.5.1 "Pseudodiffusive" transport in clean graphene nanoribbons

Transport through short and wide clean graphene monolayers around the CNP shows very peculiar behaviors analogous to those observed in diffusive coherent disordered conductors. This turns out to be a mere but extremely surprising coincidence, due to the particular distribution of the transmission coefficients. In two first theoretical studies, Tworzydło et al. [77] and Katsnelson [78] investigated the transmission coefficients for a ribbon of length $L$ and width $W$ in the limit of short and wide armchair ribbons $(L<W)$ with hardwall or smooth confining potential at the edges. The system is kept at energies around the CNP, and it is connected to two leads at high potential with a large number $N$ of active conductive channels. The electronic transmission is thus mainly sustained by tunneling through evanescent modes, and an analysis based on the Dirac equation leads to

$T_{n} \approx \frac{1}{\cosh ^{2}[\pi(n+1 / 2) L / W]} \quad$ with $n=0, \pm 1, \pm 2, \ldots$

for the hardwall confining potential, and a very similar expression for the smooth potential [77]. As a consequence, in the limit of a very large number of transmission channels and high aspect ratio $(N>>W /$ $L \rightarrow \infty)$

$$
\begin{gathered}
G=\frac{4 e^{2}}{h} \sum_{n} T_{n}=\frac{4 e^{2}}{\pi h} \frac{W}{L} \\
F=\frac{\sum_{n} T_{n}\left(1-T_{n}\right)}{\sum_{n} T_{n}}=\frac{1}{3}
\end{gathered}
$$

where $G$ is the conductance and $F$ is the Fano factor, i.e., the ratio between shot noise and Poissonian noise. In this limit, the system is ohmic with minimum conductivity $\sigma_{\min }=4 e^{2} / \pi h$ and Fano factor equal to $1 / 3$, as in the case of a diffusive conductor. This theoretical result has been tested numerically by calculations based on a tight-binding model [77, 79], and a good agreement was found, as shown in Fig. 7.

Schomerus has recently proved [80] that this

\section{量 Springer}




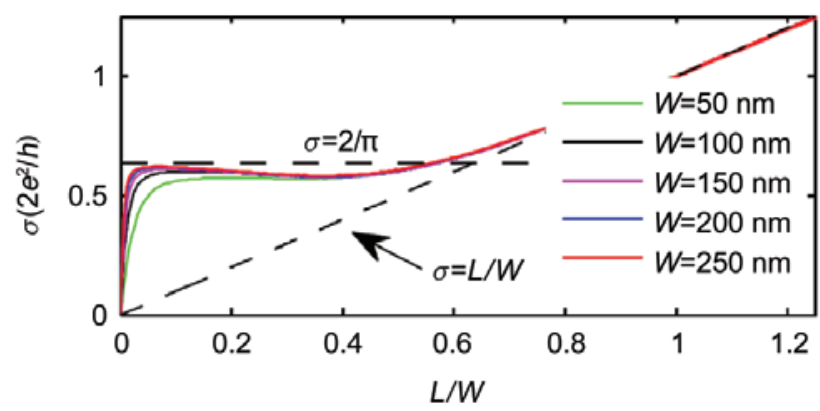

Figure 7 Conductivity $\sigma$ of a zigzag ribbon as a function of its aspect ratio (length $L$ divided by width $W$ ), in the case of graphene with leads and energy just above the charge neutrality point. For short and wide nanoribbons $(0.1<L / W<0.4)$, the value of the conductivity is very close to $4 e^{2} / \pi h$. This figure is taken from Ref. [79]

behavior is essentially independent of the specific configuration of the contacts, provided that the graphene system is very close to the neutrality point and the leads support a large number of propagation modes. The introduction of disorder is expected to affect the Fano factor, but the literature does not agree on the results. In one case [81] the Fano factor turns out to be lowered by disorder, but still retains a value close to 0.3. In another case [82], the Fano factor is found to be a little lower than $1 / 3$ for very weak disorder with a small peak at the neutrality point, and then remain around $1 / 3$ for short and wide ribbons for weak disorder, and to exceed $1 / 3$ for stronger disorder.

Three reports of recent experimental work [8385] on exfoliated graphene samples confirm the main theoretical predictions. Miao and co-workers [83] have evidenced that for wide and short graphene strips $(W / L>4)$ the minimum measured conductivity approaches the value $4 e^{2} / \pi h$. This result has been observed in particular for very broad ribbons $(W>>L)$, because in this case boundary effects are negligible. DiCarlo and co-workers [84] and Danneau and coworkers [85] performed measurements of the shot noise and observed the predicted value, $F=1 / 3$, for clean samples. Moreover, some other predictions [82] seem to be confirmed, i.e., the increasing value of the Fano factor for weakly disordered systems [85] and the presence of a (larger than expected) peak at the neutrality point for very clean ribbons [84].

The coincidence between the behavior of coherent disordered conductors and short and wide ballistic graphene ribbons is a puzzling issue, whose origin, if any, is not clearly understood.

\subsubsection{High magnetic fields and spatial chirality of currents in GNRs}

As seen in Section 1.1, a homogeneous perpendicular magnetic field $B$ that threads a $2-D$ graphene sheet induces Landau levels spaced as $\sqrt{n}$ and proportional to $\sqrt{B}[26,86]$. A prescription for the inclusion of magnetic field effects into the band structure of carbon-based nano-networks can be found in Ref. [87]. An energy structure similar to that of 2-D graphene is also observed for bulk electrons in nanoribbons, provided that the width $W$ of the ribbon is larger than the magnetic length. At the borders of the Brillouin zone, the bands bend upward or downward due to the finite width of the ribbon. The corresponding magnetic states are located at the upper and lower edges.

In the simple N-N tight-binding model described in Section 1.2, we can account for the magnetic field by means of the Peierls phase factors on the hopping parameters. By choosing the gauge properly, the Hamiltonian preserves the translation invariance along the longitudinal axis of the ribbon and can be conveniently expressed on the Bloch sums basis. Armchair and zigzag ribbons are still equivalent to a $2 N$-orbital chain and two coupled $N$ orbital chains, respectively, but the hopping energies now depend on the chain index $n=1,2, \ldots, N$ [48]. In the case of aGNRs, the coupling parameters between the two chains become (see Fig. 8(a))

$$
d_{n}(k)=\exp \left[ \pm\left(\frac{k b}{2}-\frac{n \alpha}{2}+\frac{N+1}{4} \alpha\right)\right]
$$

where $\alpha=2 \pi \Phi(B) / \Phi_{0}$ is proportional the ratio between the magnetic flux $\Phi$ through a hexagonal plaquette of the honeycomb lattice and the elementary magnetic flux $\Phi_{0}$. In the case of zGNRs, the hopping $k$-dependent parameters between the orbitals of the chain become (see Fig. 8(c))

$$
c_{n}(k)=\cos \left(\frac{k a}{2}-\frac{n \alpha}{2}+\frac{N+1}{4} \alpha\right)
$$

A direct numerical diagonalization of the Hamiltonian provides the energy bands for an $N=611$ armchair ribbon (Fig. 8 (b)) and $N=353$ zigzag ribbon (Fig. 8(d)) 


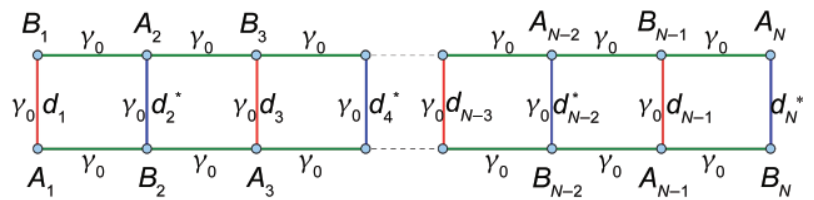

(a)

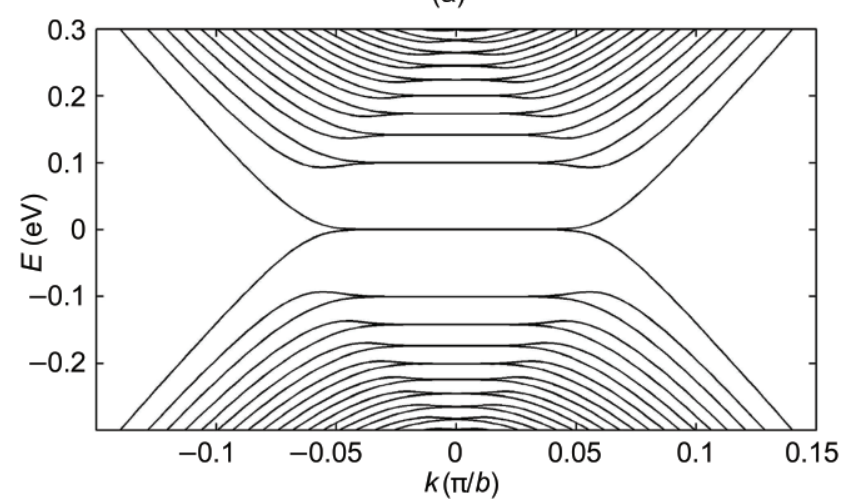

(b)

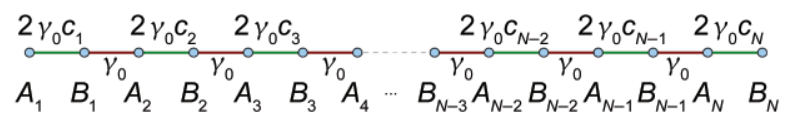

(c)

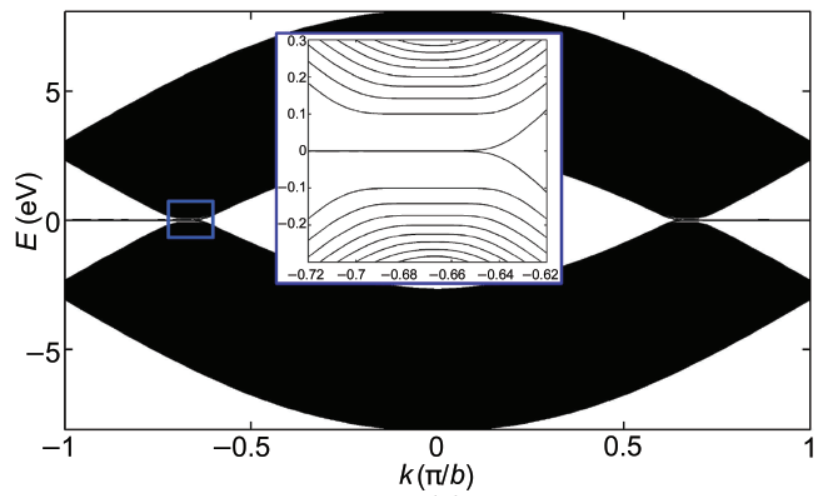

(d)

Figure 8 (a) Coupled chains equivalent to an armchair ribbon threaded by a perpendicular and homogeneous magnetic field $B$; (b) energy bands of an aGNR with $N=611$, corresponding to a width of about $75 \mathrm{~nm}$, for $B=10 \mathrm{~T}$; (c) chain equivalent to a zigzag ribbon threaded by a perpendicular and homogeneous magnetic field $B$; (d) energy bands of a $z G N R$ with $N=353$, corresponding to a width of about $75 \mathrm{~nm}$, for $B=10 \mathrm{~T}$

for $B=10 \mathrm{~T}$. We consider ribbons much wider (about $75 \mathrm{~nm}$ ) than those considered in the absence of a magnetic field, in order to have the onset of Landau levels at relatively small magnetic fields.

In the case of armchair ribbons, see Fig. 8(b), we can clearly observe the sequence of doubly degenerate Landau levels. At the borders of the Brillouin zone, the average transverse position of the states approaches the edges of the ribbon and the energy bands bend upward for electron-like particles and downward for hole-like particles. In fact, the particles cannot complete the cyclotron orbit due to the edges and as a consequence their energy rises. As in an ordinary 2-D electron gas, the states on the right side of the Brillouin zone are located on the upper edge of the ribbon, while the states on the left side are located on the lower edge. The double degeneracy of the bands is removed for edge states. This can be explained in terms of the Dirac equation since the boundary conditions for armchair ribbons entail the admixing of the valleys [88]. As a consequence, the corresponding wavefunctions hybridize thus giving rise to the observed structure.

In the case of zigzag ribbons, see Fig. 8(d), we observe a positive and a negative set of Landau levels around each of the two valleys, and a zero energy level that extends from one valley to the other. The states on the right side of each valley are located on the upper edge of the ribbon, while the states on the left side of each valley are located on the lower edge. Again, when the average transverse position of the states approaches the edges of the ribbon, the energy bands bend upward for electron-like particles and downward for hole-like particles. The structure of the energy bands around the two valleys is exactly the same. Again, this can be understood by considering the Dirac equation. In contrast to armchair ribbons, the boundary conditions for the wavefunction do not mix the two valleys and therefore, close to the CNP, they behave independently of each other. The Dirac equation also explains the peculiar structure of the two lowest Landau levels. The corresponding wavefunctions are located at one edge for a sublattice and behave as ordinary magnetic states for the other.

From the transport perspective, the first important consequence of the magnetic electronic structure of GNRs is the theoretical prediction and the experimental observation of the so called anomalous (half-integer) Hall effect [7, 31, 89, 90], i.e., the magnetoconductivity is given by

$$
G=\frac{4 e^{2}}{h}\left(n+\frac{1}{2}\right)
$$

The large separation between the first Landau levels allows the observation of this phenomenon at room temperature. 
In addition to this, magnetic edge states have peculiar and interesting properties. Since the direction of the carrier group velocity depends on the sign of their energy band slope with respect to the wavevector $k$, electron-like particles can only flow from the left side of the ribbon to the right side along the upper edge, and from the right side to the left side along the lower edge. In the case of holelike particles the energy bands are reversed and they can only flow from the left side of the ribbon to the right side along the lower edge, and from the right side to the left side along the upper edge. The spatial separation between the conductive channels that support current in opposite directions is called spatial chirality. In the case of GNRs, electron- and hole-like particles have opposite spatial chiralities [48]. This property may be exploited to manipulate the current and obtain a current switch [91]. In fact, the edge the transport currents flow along is determined by the position of the energy of the electrons with respect to the CNP, and their energy can be varied by means of top or back gates, even locally.

\section{Disorder effects}

Transport properties in graphene-based materials also turn out to be strongly affected by disorder, which can originate from impurities (charges trapped in the oxide, chemical impurities, etc.), topological defects (vacancies, edge disorder...), or long range deformation modes (ripples) in 2-D graphene. A certain control on contamination can be obtained by annealing processes at high temperature in ultrahigh vacuum, or, at low temperature, by current induced cleaning [92]. Importantly, the dominant scattering processes and resulting transport features are very dependent on the range of the disorder potential and the robustness or destruction of the underlying sublattice symmetries.

For massless Dirac fermions, a long range scattering potential, i.e., with Fourier components $V(\boldsymbol{q})$ such that $|\boldsymbol{q}|<<|\boldsymbol{K}|$, will strongly reduce the intervalley scattering probability between the two non-equivalent Dirac nodes $\left(\boldsymbol{K}_{-} \rightarrow \boldsymbol{K}_{+}\right)$. In the onedimensional case provided by armchair CNTs, this results in a full suppression of backscattering probability as demonstrated by Ando and co-workers $[93,94]$. The impact of long range disorder in twodimensional graphene remains more controversial, with open issues concerning how weak or strong localization regimes are genuinely affected by the specific properties of Dirac fermions [95, 98].

In contrast, the presence of short range disorder $(q \approx k)$ will allow for all possibilities of intravalley and intervalley scattering events between $K_{+}$and $K_{-}$, leading to stronger backscattering and localization effects, although the possibility of a true Anderson localization in two-dimensional graphene remains fiercely debated theoretically, because of the "Dirac nature" of low energy excitations $[99,101]$.

Disorder effects in the quantum coherent regime can yield localization regimes [102-105], and indeed some experimental evidence for weak and strong Anderson localization regimes has been reported in disordered CNTs [4, 106-115], but one should also stress that the effects of disorder on quantum transport in graphene-based materials of lower dimensionality, such as CNTs and GNRs, are expected to be maximized compared to the case of 2-D graphene. Indeed, low dimensionality and confinement effects yield strong modifications of band structures with the appearance of van Hove singularities, close to which energy dispersion-or wavepacket velocity - is very low. In the forthcoming sections, we will focus on short range disorder effects by using the Anderson-type disorder potential, and we will review their effects on electronic states, mean free paths and localization phenomena.

\subsection{Density of states in weak disordered CNTs and GNRs}

The spectrum of a system may be affected by weak disorder only through small energy shifts in the energy. A constant DoS will therefore remain unaffected by weak disorder. Regions of slowly changing DoS will only show small effects. Discontinuities and van Hove singularities will, in contrast, be strongly affected and appear smeared out at the energy scale of the disorder strength [116, 117].

The DoS of a general quantum wire under the influence of Anderson disorder can be obtained via an algorithm based on diagrammatic perturbation 


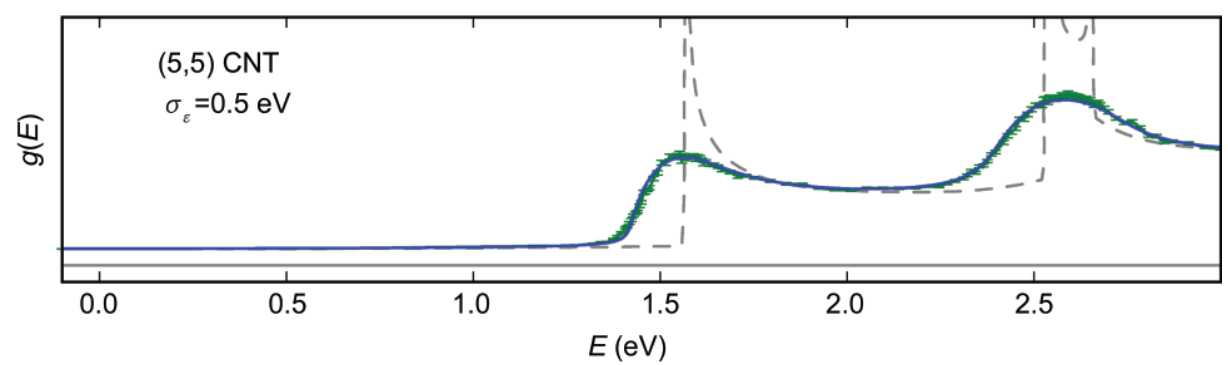

Figure 9 Density of states $g(E)$ of an infinite armchair $(5,5)$ CNT under the influence of Anderson disorder. Dashed line ( $g r e y): ~ g(E)$ of the clean system displaying the van Hove singularities. Solid line (blue): $g(E)$ from Eq. (44). Data with error bars (green): values obtained numerically by sampleaveraging. Adapted from Ref. [117]

theory that takes into account localization effects by including multiple scattering [116]. By dropping the crossing diagrams within the noncrossing approximation (NCA) [118], we can write the selfenergy $\sum(E)$ to all orders as a recursive expression, which can then be iterated numerically until selfconsistency is reached. Though the applicability of the NCA is not obvious, it can be justified by comparing the contribution of various terms at low orders [116].

The original formulation of this approach is restricted to the special case of CNTs where all atoms are equivalent through symmetry and the self-energy takes the same value for all atoms. It can, however, be generalized to arbitrarily structured quantum wires using matrix notation. The self-energy $\sum(E)$ that accounts for disorder is a diagonal matrix obeying the recursive relation,

$$
\left[\sum(E)\right]_{i, j}=\delta_{i j} \sigma_{\varepsilon i}^{2}\left[\left(E+\mathrm{i} 0^{+}-H_{0}-\sum(E)\right)^{-11}\right]_{i, j}
$$

For a periodic system, the self-energy has the same periodicity as the Hamiltonian. The blocktridiagonal matrix $\left(E+\mathrm{i}^{+}-H_{0}-\sum(E)\right)$ can therefore be inverted numerically using a highly convergent renormalization-decimation algorithm [119, 120], allowing us to go beyond the energy range near the Fermi energy, where the special band structure allows analytic inversion.

Starting with $\sum=0$, each numerical iteration of this recursive relation is equivalent to one additional perturbative order. Typically, convergence is achieved after less than ten iterations, except for energies near a van Hove singularity, where hundreds of iterations may be necessary. This clearly indicates that low order perturbation theory breaks down near band edges.
The local DoS (LDoS) of each orbital $i$ in the unit cell can now be obtained directly from the imaginary part of the Green's function,

$$
g_{i}(E)=-\frac{1}{\pi} \operatorname{Im}\left[\left(E+\mathrm{i} 0^{+}-H_{0}-\sum(E)\right)^{-1}\right]_{i, i}
$$

Figure 9 shows this quantity in direct comparison with the numerically exact value obtained by sample averaging. The slight deviation visible at the flanks of the van Hove singularities is caused by the NCA [116]. The elastic mean free path $l_{\mathrm{el}}$ based on the DoS of a disordered system, displayed in Fig. 10, is no longer a purely perturbative quantity, but takes into account the scattering into localized states present at any given energy.

The DoS of aGNRs, see Fig. 11(a), is obtained straightforwardly and shows the expected smoothing of van Hove singularities (including those at the gap edges) in complete analogy to CNTs. For zGNRs (Fig. 11(b)), the most prominent feature in the DoS is the strongly broadened edge state around the Fermi energy, whose shape displays a clear deviation of the NCA from the numerical exact calculation.

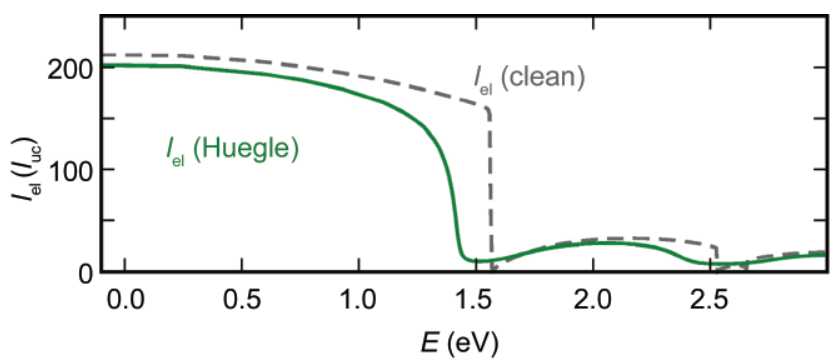

Figure 10 The elastic mean free path $I_{\mathrm{el}}$ in a $(5,5)$ CNT with Anderson disorder of strength $\sigma_{\varepsilon}=0.5 \mathrm{eV}$. Both lines are obtained from Eq. (49). In the case of the dashed line, $g(E)$ and $N_{\text {ch }}$ correspond to the values of the clean system, resulting in discontinuities at the band edges. For the solid line, the disorder effects on $g(E)$ and $N_{c h}$ are taken into account 

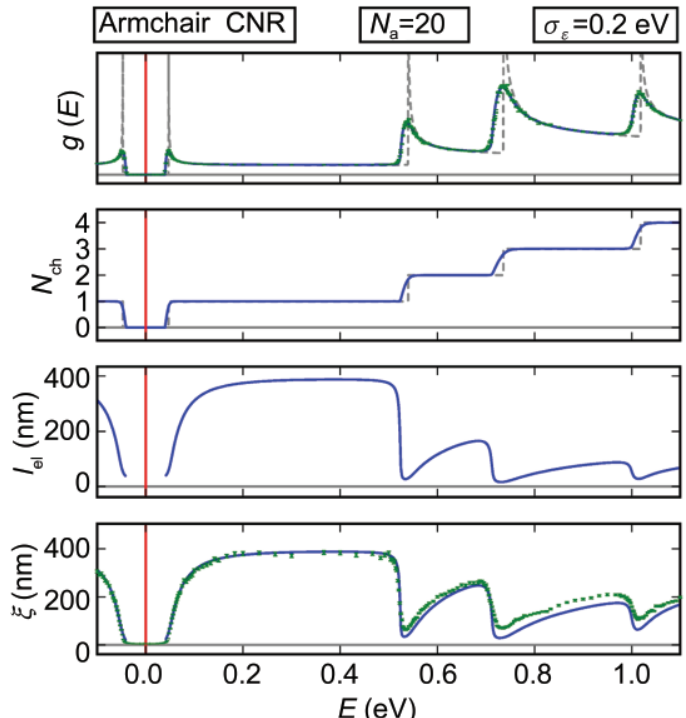

(a)
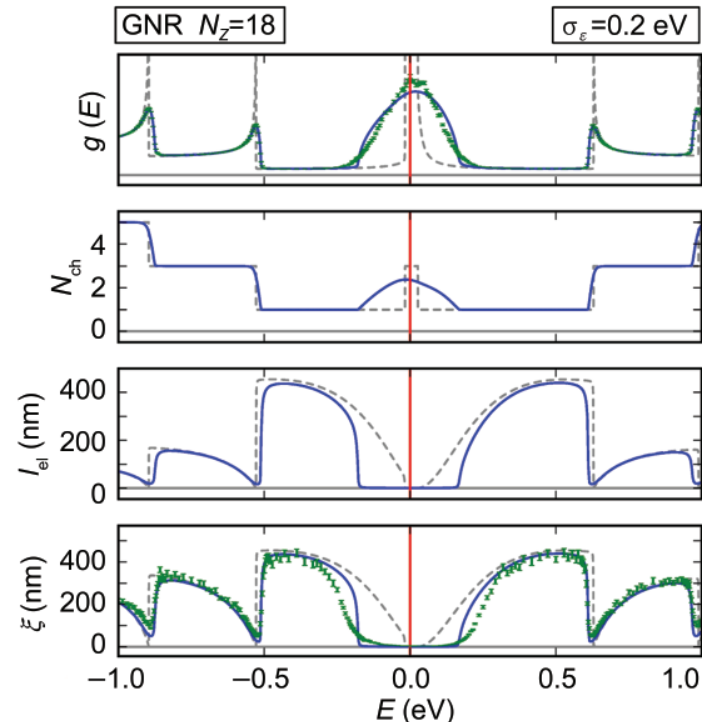

(b)

Figure 11 (a) Density of states $g(E)$, number of channels $N_{\mathrm{ch}}$, elastic mean free path $I_{\mathrm{el}}$ and localization length $I_{\text {loc }}$ of an armchair GNR of width $N_{\mathrm{a}}=20$ under the influence of Anderson disorder ( $\sigma_{\varepsilon}=0.2 \mathrm{eV}$ ). Dashed lines ( $\left.g r e y\right): ~ g(E)$ and $N_{c h}$ of the clean system. Solid lines (blue): $g(E)$ from Eq. (44), $N_{\text {ch }}$ obtained as transmission through a cross section through an infinite system with self-energy from Eq. (43) and lengths obtained from these. Data with error bars (green): values obtained numerically by averaging over about 180 samples of length to 20000/uc. (b) The same for a zigzag GNR of width $N_{z}=18$. The most prominent feature is the disorder-broadened peak in the Dos caused by the edge state that causes a strong reduction in the elastic mean free path and the localization length. The analytical values fail to describe the true shape of this feature because the expressions neglect the difference in the various channels. Adapted from Ref. [117]

\subsection{Elastic mean free paths}

The elastic mean free path $\left(l_{\mathrm{el}}\right)$ is a key quantity in mesoscopic transport. Hereafter we focus on the case of short range disorder, which allows us to illustrate common properties of transport length scales in graphene-based low dimensional materials. The Anderson disorder model is the most generic case for investigating localization phenomena in low dimensions. In this model, the onsite energies of $\mathrm{p}_{z}$ orbitals assume random values within an interval $(-W / 2, W / 2)$ with a given probability distribution. Hereafter we assume a uniform probability distribution i.e., $P=1 / W$.

In a situation of weak disorder, within the Born approximation scheme, $l_{\mathrm{el}}$ can be derived at a certain degree of approximation, thus enabling the possibility to extract an analytical expression. The simplest approximation for 2-D graphene can be derived as follows. The total DoS can be written as

$$
\rho(E)=\left(\sqrt{3} a^{2} / 2 \pi\right)|E| /\left(\hbar v_{\mathrm{F}}\right)^{2}
$$

As a result, writing $l_{\mathrm{el}}=v_{\mathrm{F}} \tau$, and using a simple Fermi Golden Rule approach for the elastic scattering time $\tau\left(\tau^{-1}=(2 \pi / \hbar) \rho\left(E_{\mathrm{F}}\right) W^{2} / 12\right)$, we obtain

$$
l_{\mathrm{el}} \approx\left(\gamma_{0} / W\right)^{2} \alpha\left|\gamma_{0}\right| /|E|
$$

which diverges when $|E| \rightarrow 0$. This crude estimation pinpoints a difficulty in calculating transport length scales when the Fermi level lies close to the Dirac point. A numerical calculation within the Kubo approach allows the evaluation of $l_{\mathrm{el}}$ at a quantitative level in 2-D disordered graphene with Anderson scattering potential. In Fig. 19 (inset) (adapted from Ref. [152]), $l_{\mathrm{el}}$ is shown for $W=1,1.5,2,2.5$ in $\gamma_{0} / 2$ units and ranges from several tens of nanometers down to a few nanometers close to the Dirac point (for $W \approx 3.4 \mathrm{eV}$ ).

In quasi-1-D systems (such as CNTs and GNRs), scattering angles are restricted to two cases. Forward scattering events at zero angle lead to momentum relaxation but do not affect the elastic transport length scale. Only the backscattering events at an angle of $\pi$ are taken into account in the derivation of the elastic mean free path $l_{\mathrm{el}}$. By using the Anderson disorder model, White and Todorov derived an analytical formula for the elastic mean free path 
$\left(l_{\mathrm{el}}\right)$ close to the CNP [94]. For armchair metallic nanotubes, they obtained

$$
l_{\mathrm{el}}=18 \sqrt{3} a_{\mathrm{cc}}\left(\gamma_{0} / W\right)^{2} \mathrm{~N}
$$

showing that, for a fixed disorder strength, $l_{\mathrm{el}}$ will increase linearly with the nanotube diameter, a property unique to these systems and pinpointing long ballistic systems. Triozon and co-workers [122] numerically confirmed such a prediction and further reported the strong energy dependence of $l_{\mathrm{el}}$ close to the onsets of new subbands. Similarly, in metallic $N$-aGNRs [36] $l_{\mathrm{el}}$ was derived as

$$
l_{\mathrm{el}}=12\left(\gamma_{0} / W\right)^{2}(N+1) a_{\mathrm{cc}}
$$

Therefore, both low dimensional carbon systems show a mean free path that diverges with increasing diameter or ribbon width for a fixed disorder strength $W$. Note however that only armchair nanotubes really display 1-D massless Dirac fermions close to the CNP, since there is a gap opening in all GNRs due to edge boundary conditions.

An expression for $l_{e l}$ that holds at arbitrary energies can be derived from the Fermi Golden Rule [117]

$$
l_{\mathrm{el}}=l_{\mathrm{uc}} N_{\mathrm{ch}}\left[\pi^{2} \sum_{i}^{\mathrm{uc}} \sigma_{\varepsilon i}^{2} g_{i}^{2}(E)\right]^{-1}
$$

with $l_{\text {uc }}$ the length of the unit cell and the sum running over all orbitals $i$ within one unit cell. In this form, the expression can be applied to arbitrary quantum wires, including GNRs, where it also covers the special case of edge disorder by making $\sigma_{\varepsilon i}^{2}$ dependent on the orbital number $i$.

Neglecting multiple scattering, the elastic mean free path $l_{\mathrm{el}}$ and the diffusive transmission $T_{\text {diff }}$ are defined in terms of the $\operatorname{LDoS} g_{i}(E)$ of the disorder-free system. Likewise, $N_{c h}$ is defined by the leads, where it follows an exact integer step function. Near band edges, this diffusive transmission is discontinuous, as can be confirmed numerically to arbitrary precision, by computing it as the sample average $\langle T\rangle$ of the transmission of many disorder configurations, as displayed in Fig. 12.

For stronger disorder and extended disordered regions, the elastic mean free path is no longer a purely perturbative quantity due to the fact that it depends on the DoS and has to include non-

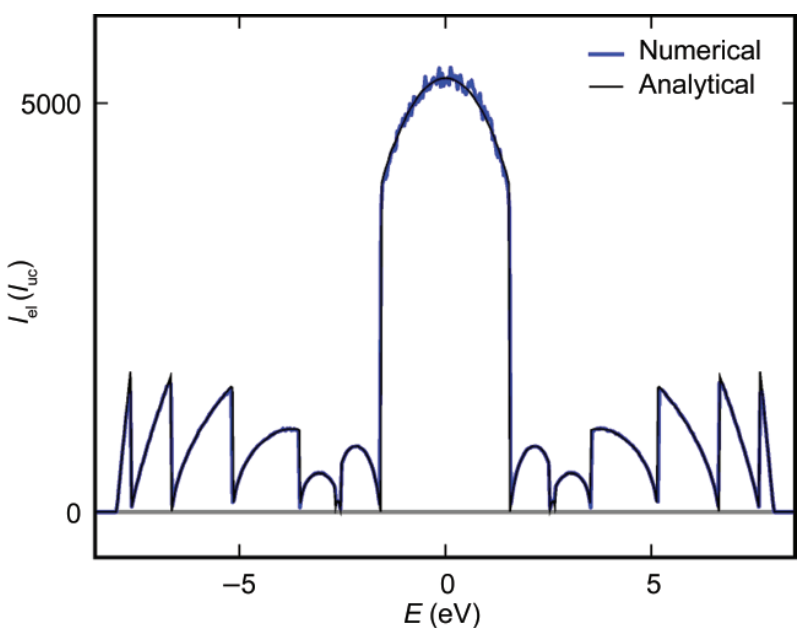

Figure 12 The elastic mean free path $I_{\mathrm{el}}$ in a $(5,5)$ CNT with Anderson disorder of strength $\sigma_{\varepsilon}=0.1 \mathrm{eV}$. The numerical results are obtained from the transmission of a single disordered unit cell, averaged over 4000 samples as $l_{\mathrm{e}}=L /\left(N_{\mathrm{ch}} /\langle T\rangle-1\right)$. The analytical result is obtained via Eq. (49) using $g(E)$ of a disorder-free system

perturbative effects near van Hove singularities. We can, however, retain Eq. (49) by simply including the non-perturbative effects in $g_{i}(E)$ using Eq. (44). Furthermore, the number of channels $N_{\mathrm{ch}}$ must also take into account the non-perturbative effects near band edges, which can be achieved by including the self-energy term Eq. (43) in the calculation of the transmission through a cross section of an infinite quantum wire as described in Ref. [117]. Incorporating these effects, we obtain the elastic mean free path in an infinitely long disordered quantum wire as displayed in Fig. 10.

\subsection{Quantum interference effects and localization phenomena in disordered graphene-based materials}

Knowledge of the mean free path $l_{\mathrm{el}}$ in quasi-1-D systems is crucial since it allows the identification of the frontier between the ballistic and the diffusive propagation of wavepackets in weakly disordered systems. Assuming that the transport regime remains coherent, a new class of scattering paths will yield an important contribution to the resistance, known as the weak localization correction, which eventually turns the metallic state to an insulating one [102105]. The localization length $\xi$ is the other physical length scale that defines such a transition, where the conductance decays exponentially with the system length as $G \approx G_{0} \exp (-L / \xi)$. 
Weak localization phenomena have been observed in multiwalled CNTs with diameters ranging from 3-20 nm [106, 107, 110-113]. Similarly, weak localization has been recently clearly unveiled in GNRs with widths in the order of $200-500 \mathrm{~nm}$ [123]. In addition, a transition to weak anti-localization has been reported [14, 123, 124]. Weak anti-localization (WAL) in graphene-based materials is argued to originate from some pseudospin-induced sign change of the quantum correction, similar to what is observed in systems with strong spin-orbit coupling [95-98]. For the same kind of disorder potential, pseudospin symmetries might not be preserved for GNRs with small width. We can expect that the effect of edge disorder and intrinsic defects (topological, vacancies, adsorbed impurities, ...) will play an increasing role as GNR width decays from $\sim 20 \mathrm{~nm}$ down to $\sim 5 \mathrm{~nm}$.

It is thus genuinely important to evaluate the varying effects of disorder on quantum transport as the dimensionality or symmetries are changed. It is worth stressing that, in order to unveil weak localization effects, an external magnetic field is generally applied to tune the intensity of quantum corrections. Indeed, the phase of the quantum wavefunction is modified by the magnetic field (through a term giving the circulation of the potential vector along the scattering path), which reduces the probability of return to the origin and enhances the conductance (this phenomenon is known as the negative magnetoresistance effect). However, for low dimensional systems such as CNTs, it was demonstrated that a magnetic field has also severe consequences on the band structure, so that the resulting magnetofingerprints in localization regimes become more complicated [106, 107, 110-113] to follow. This will also apply to magnetoresistance effects in GNRs with width $\leqslant 10 \mathrm{~nm}$.

Let us illustrate the quantum localization effects in GNRs. As seen in Section 1.2, zGNRs display very peculiar electronic properties, with wavefunctions sharply localized along the ribbon edges for energies in the vicinity of the CNP. Using a conventional Landauer-Büttiker approach [36-38, 125], one can explore the scaling properties of the quantum conductance of these systems. In Fig. 13, the energy- dependent conductance for both zGNRs and aGNRs of width $\sim 20 \mathrm{~nm}$ are shown for pure, weak disorder $(W=0.5)$ and strong disorder $(W=2)$ limits, respectively.

In the weak disorder limit ( $W=0.5$, inset of Fig. 13(a)), aGNRs appear to be much less sensitive to disorder effects than zGNRs with the same width. As can be seen in the insets of Figs. 13(a) and (b), the averaged normalized conductances are exponentially damped, following $\left\langle\ln G / G_{0}\right\rangle \approx L / \xi$, where an average over $\sim 400$ different disorder configurations

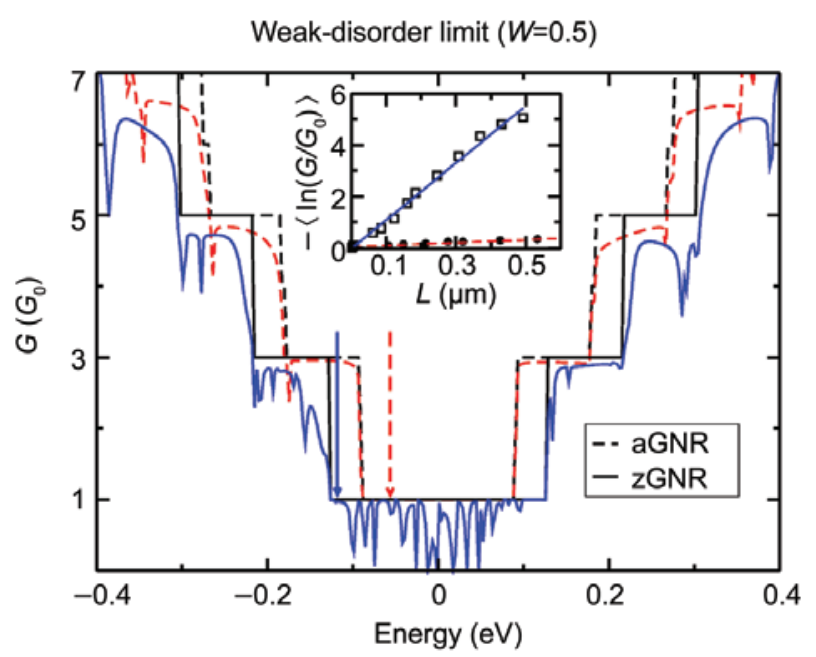

(a)

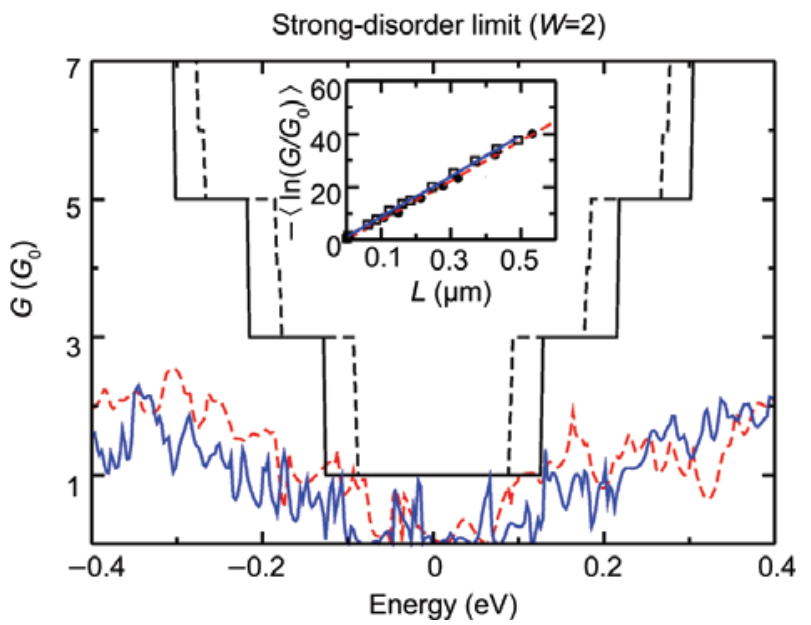

(b)

Figure 13 (a) Conductance for a single disorder configuration of a zigzag (solid blue line) and an armchair (dashed red line) GNR with width $\approx 20 \mathrm{~nm}$ for $W=0.5$. Black lines correspond to ideal zigzag (solid lines) and armchair (dashed lines) ribbons. Inset (a) Configuration averaged (over 400 samples) normalized conductance as a function of GNR length for both zigzag and armchair GNRs. The solid blue (dashed red) arrow shows the energy at which the calculations for the zGNR (aGNR) were performed. (b) The same information as for (a) but for a larger disorder strength ( $W=2)$. Adapted from Ref. [152] 
has been performed. From these calculations, $\xi$ is found to be up to two orders of magnitude smaller in zigzag than in armchair ribbons in the low disorder limit $(W=0.5)$, for an energy value far from the close vicinity of the CNP (following [36-38]). In contrast, for disorder strength as large as $W=2$ (inset of Fig. 13 (b)), the localization lengths for both types of ribbons are almost equal, showing that edge symmetry does not play any role. This result can be understood by the lower transport dimensionality in the case of zigzag edge symmetry, driven by more confined wavefunctions [27-29, 31, 33, 35].

An important result of mesoscopic physics is that there exists a fundamental relationship between $l_{\mathrm{e}}$ and $\xi$ known as the Thouless relation [126]. In a strictly 1-D system, Thouless [126] demonstrated that

$$
\xi=2 l_{\mathrm{el}}
$$

whereas for quasi-1-D systems (with $N_{\perp}(E)$ conducting channels), the relation was generalized as

$$
\xi(E)=\left[\beta\left(N_{\perp}(E)-1\right) / 2+1\right] l_{\mathrm{el}}(E)
$$

with $\beta$ a factor dependent on the time-reversal symmetry [127]. Avriller and co-workers [128] recently confirmed numerically such relation by studying chemically doped metallic CNTs.

As was shown in Ref. [117], Eq. (51) still holds to good precision near van Hove singularities on the condition that the non-perturbative effects of multiple scattering are correctly included in the calculation of the DoS and the number of channels $N_{\perp}$ as described before for the elastic mean free path. As can be seen in Fig. 14, this correction greatly improves the agreement with the true value obtained via sampleaveraging. The remaining deviation is predominantly caused by the fact that the different conduction channels have very different velocities and thereby very different elastic mean free paths, whereas Eq. (51) is based upon the assumption of equivalent channels with one common elastic mean free path.

The expressions for the DoS and the localization length obtained and demonstrated before for CNTs are sufficiently general to apply to GNRs as well. The data in Fig. 11(a) demonstrate that the values obtained analytically do indeed match the results of numerical sample averaging. For semiconducting CNTs and GNRs, the behavior near the gap is correctly reproduced, showing a slight disorderinduced reduction of the gap width.

As can be seen in Fig. 11(b), the expressions still hold for zGNRs over most of the energy spectrum, and even qualitatively describe the effects of the edge state around the Fermi energy: the extremely high DoS of the Fermi energy is smeared out in the energy range and the localization length is drastically reduced not only in the narrow region of the lowdispersive edge state itself, but-due to the disorderinduced broadening in energy - in an extensive region around the Fermi energy. However, the expressions fail to describe the actual shape of the energy-broadened peak in the DoS, as well as the flanks of the suppressed region in the localization length. These strong deviations can be traced to the assumption of equivalent conduction channels that is made in Eq. (51). Near the edge state, this assumption fails completely.

\subsection{Edge disorder in GNRs}

In contrast to two-dimensional graphene and CNTs, GNRs are subject to chemical passivation and roughness at the edges. In some cases, the nature of the chemical groups that passivate the edges (usually

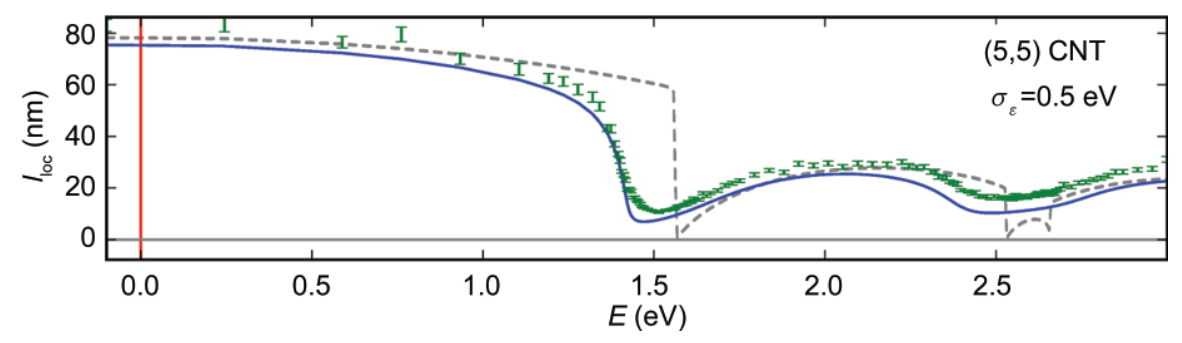

Figure 14 Localization length of a $(5,5)$ CNT. Dashed line (grey): value obtained from Eq. (51) by naively using the perturbative values for $l_{\mathrm{el}}$ and $N_{\perp}$ based on the self-energy and DoS of the disorder-free system. Solid line (blue): value based on the non-perturbative quantities including disorder in the self-energy and DoS. The numerical values indicated by error bars (green) are obtained from logarithmic sample averaging. Adapted from Ref. [117] 
H) can be determined experimentally, thus enabling a certain control of the ribbon. Unfortunately, spatial regularity of the edges is much more difficult to achieve and state of the art etching techniques cannot avoid roughness. Direct or indirect evidence of edge disorder has been observed in many different graphene samples, independently of the technique exploited to fabricate them.

In the literature, the edges of graphene sheets from highly oriented pyrolitic graphite have been directly investigated by scanning tunneling microscopy (STM) in association with scanning tunneling spectroscopy (STS) [129-132] and by direct contact atomic force microscopy (AFM) [133, 134]. Micro-Raman spectroscopy has also proved to be a valuable tool for studying the armchair or zigzag orientation of the ribbon edges locally [135]. In all these experiments, the structure of the edges turned out to be very irregular, with alternation and mixing of zigzag and armchair terminations, protrusions and dents or more complex structures. In general, armchair segments are considerably longer than zigzag fragments, thus evidencing a lower stability for the latter. The measurement of the transport properties of lithographically etched ribbons $[18,19]$ also provides indirect evidence of the edge roughness. In particular, hints of inactive edge regions and dependence of the maximum resistivity on the ribbon width have been related to a possible disorder on the edges.

Similar conclusions have been drawn for ultrathin epitaxial graphene grown on silicon carbide crystals $[6,136]$. In this case, an indication of edge disorder (due to roughness or chemisorbed molecules) comes from the lower than expected number of conductive modes, due to inactive edge regions, and from the behavior of weak localization.

Innovative techniques for fabrication and etching of graphene ribbons have been shown to reduce the edge irregularities considerably, besides allowing the realization of ultranarrow (few $\mathrm{nm}$ ) structures. The chemical technique developed by Dai and coworkers [24] enables formation and selection of long and ultrasmooth nanoribbons. Despite the high spatial regularity, field-effect transistors based on these systems [137] have shown a non-negligible scattering related to disorder at edges. The very recent STM lithographic technique developed by Tapasztó and co-workers [138], allows the patterning of ultranarrow structures with the possibility of choosing orientation and width with almost atomic precision. Nevertheless, STM measurements on ribbons with a width of a few $\mathrm{nm}$ have revealed irregular oscillations in the electronic DoS, thus suggesting the possible presence of edge disorder and its importance for very narrow ribbons.

Several different edge disorder models for GNRs have been proposed in the literature. The basic idea is to start from a tight-binding Hamiltonian for a clean and regular ribbon (in general with hydrogen passivation on the edges), and then adding [37] or removing [36, 139-141] carbon atoms at the edges, or varying the width of the system [142] to account for roughness, or introducing Anderson disorder [143]. The most proper way of adding/removing atoms at the edges avoids final configurations that might cause steric problems. In practice, only $\mathrm{H}-\mathrm{C}-\mathrm{C}-\mathrm{H}$ groups can be removed or added at the edges of an armchair ribbon, while particular care must be adopted when the disorder goes deeper than the first row in zigzag ribbons.

The transport properties of metallic aGNRs have been investigated in the presence of both roughness [36, 141] and Anderson disorder [143] on the edges. In the case of vacancies on the edges, the differential conductance of the system is considerably reduced and even a weak disorder on the two external rows induces a localization process, see Fig. 15(a). The localization length turns out to be particularly low around the neutrality point even for $5 \%$ of vacancies. This is attributed to a small gap opening as a result of the crossing of the two bands around $E=0$ [36] and whose width is inversely proportional to the width of the ribbon [141]. By increasing the level of disorder on the two most external rows, the system can be envisioned as a sequence of metallic and semiconducting fragments and the localization length decreases considerably within the energy range that corresponds to the semiconductor gap, see Fig. 16. Even outside this region, the conduction ability is seriously jeopardized, with a localization length of few tens of $\mathrm{nm}$. Similar results have been also recently obtained by Evaldsson et al. [140]. 


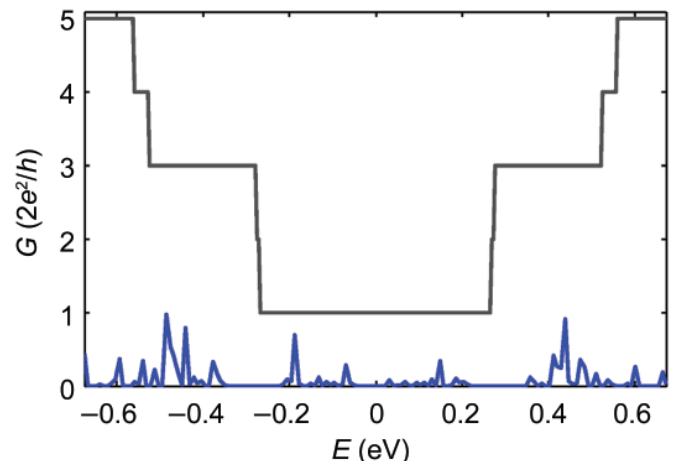

(a)

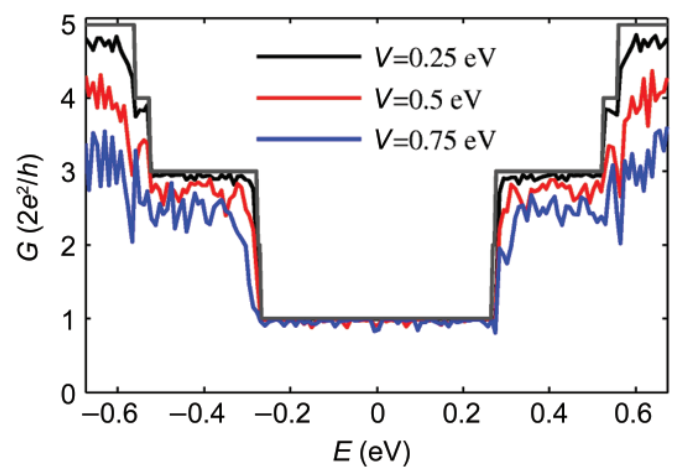

(b)

Figure 15 (a) Differential conductance of a metallic armchair ribbon $(N=53)$ with $\sim 10 \%$ of vacancies on the edges. Couples of atoms have been removed on the most external rows over a length of about $1 \mu \mathrm{m}$. For the calculation, we adopted a simple tight-binding Hamiltonian with zero site energy and hopping energy $\gamma_{0}=-2.7 \mathrm{eV}$. (b) Differential conductance for the same system with Anderson disorder on the edges. The energy of the edge sites varies randomly in the range $\pm V$ with $V=0.25,0.5$ and $0.75 \mathrm{eV}$ over a length of about $1 \mu \mathrm{m}$

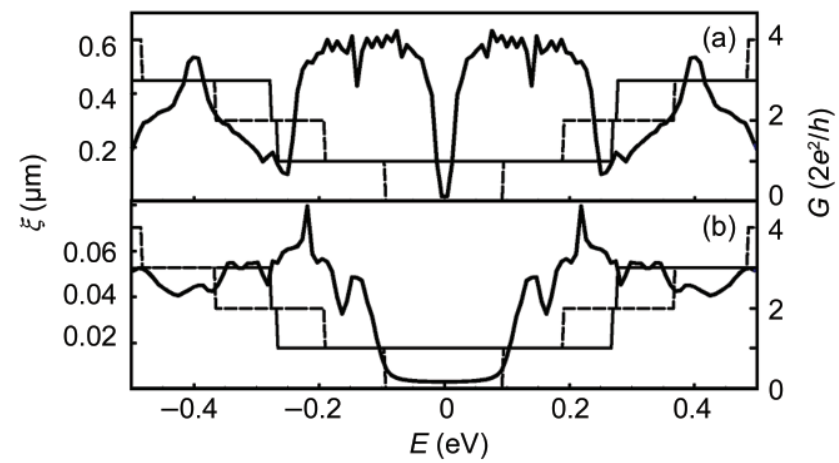

Figure 16 One half the amplitude of the localization length for a metallic aGNR with $\mathrm{N}=53$ for $5 \%$ (a) and $50 \%$ (b) concentrations of defects at the edges. Taken from Ref. [36]

The effect of sequences of large conducting and semiconductor armchair fragments has been investigated in detail by Martin et al. [142]. The length of each metallic fragment is such as to preserve the band structure of the corresponding infinitely extended ribbon. From this perspective, it is possible to obtain an effective tight-binding Hamiltonian, where the eigenfunctions of the metallic fragment play the role of "orbitals", with equispaced site energies, and the hopping energies between two subsequent fragments are related to the properties of the semiconductor fragment in between. The result is a one-dimensional impurity band insulator with conductivity

$$
\sigma \approx \mathrm{e}^{-2 \sqrt{\alpha E_{g} / T}} \text { for } T<T^{*}
$$

where $L_{\mathrm{av}}>W$ is the average length of the metallic grains, $E_{\mathrm{g}}$ is the energy gap of the semiconductor fragments, $T$ is the temperature, $T^{*} \approx\left|\gamma_{0}\right| D / L_{\text {av }}^{2}$ (with $D$ the width of the ribbon) and $\alpha$ is a numerical coefficient of the order of 1.

In the case of weak Anderson disorder on the edges, the conductance of metallic aGNRs is only slightly affected, in particular within the energy region that corresponds to a single channel, see Fig. 15(b). This result can be explained by considering the high kinetic energy of the states around the CNP [143], which are therefore scarcely affected by potential fluctuations. Just to give an idea of the low sensitivity of these states to disorder, let us consider a constant potential on both edges. It turns out that the structures of the highest valence band and the lower conduction bands do not change around the $\mathrm{CNP}$, apart from a small shift in energy and the rising of an energy gap whose width is always small and considerably suppressed for larger ribbons, see Fig. 17. The linear energy dispersion is thus preserved and the gap is almost negligible because the states that correspond to the bands around the neutrality point are spread all over the section of the system and thus the effect of the edge potential is weakened by averaging over the chains.

The two types of disorder, always confined to the two outer rows of the metallic aGNRs, have different consequences. However, there is no inconsistency in this, since the nature of the perturbation is completely different in the two cases. Roughness tends to introduce semiconductor islands and then a gap. Weak Anderson disorder does not perturb the structure of the energy bands close to the neutrality point, and therefore the backscattering is limited, at 


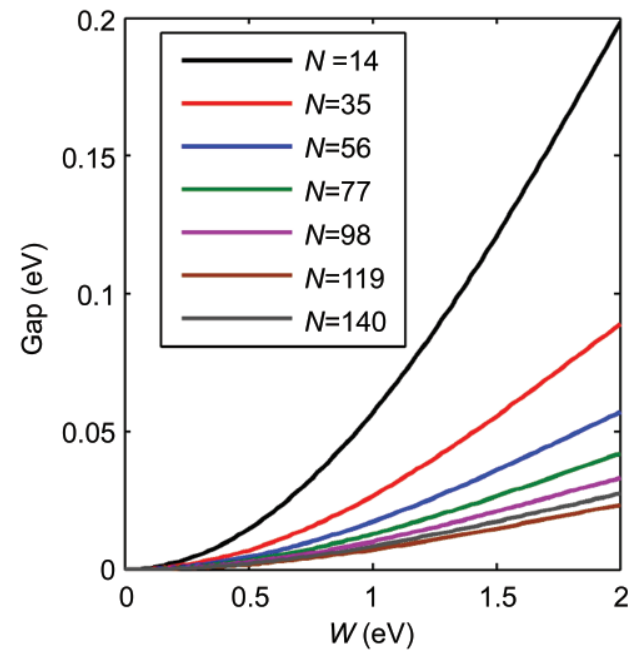

Figure 17 Gap between the highest valence band and the lowest conduction band in the presence of a constant potential $W$ on the two edges of the armchair ribbon for $N=14,35,56,77,98,119$, and 140. The larger is the strip, the smaller is the gap

least for wide ribbons.

In the case of roughness for semiconductor aGNRs, the system goes toward a strong localization regime, as in the case of metallic ribbons. However, the disorder induces states in the region of the energy gap [37, 139], thus allowing current tunneling for not very long systems. Therefore, a compromise between the width and the length of the ribbon must be found in order to retain the semiconductor behavior of the strip. In particular, the ribbon must be short enough to preserve the extension of the states just outside the gap all over its length and long enough to prevent tunneling through the states induced within the energy gap. As reported at the end of the section, this can considerably affect the $I_{\text {on }} / I_{\text {off }}$ ratio for GNRbased field-effect transistors [144-146].

In the case of zigzag ribbons, the almost dispersionless edge states around the neutrality point play a key role in determining the properties of the disordered system. In the literature, different behaviors have been observed depending on the specific model of edge disorder, with results not always consistent. Areshkin and co-workers [36] have found that $\mathrm{zGNRs}$ are much less sensitive to edge disorder than aGNRs, especially in the energy region where only one conductive mode is active. For reasonably wide strips and for an erosion of $50 \%$ $\mathrm{C}$ atoms on the eight outer rows, the localization length turns out to be of the order of $10 \mu \mathrm{m}$ (at least ten times the value for an aGNR with a much weaker disorder). To explain this, they consider that the states within the first conductive channel can be divided into two groups: almost dispersionless edge states within a very small energy range around the neutrality point $E=0$ and bulk states with energy outside this range. The width of the range is determined by the transverse width of the ribbons. The effect of roughness is mostly on the edge states, and this explains why the bulk states are not significantly affected by disorder. On the other hand, the conductance is expected to be much depressed around the neutrality point, where the effect of disorder is larger. This is only partially observed in the simulations [36], where the conductance seems to have a strong resistance to roughness compared with to the armchair case.

A rather different conclusion was obtained by Querlioz and co-workers [139], who evaluated and analyzed the wavefunction and the DoS of zigzag ribbons. From these data, the mobility edge was extracted. Their conclusion was that no ribbon has zero mobility edge and that the resistance to edge disorder for zGNRs vanishes as soon as more than one edge row is eroded. In this case, the roughness induces an Anderson insulator behavior independently of the zigzag or armchair orientation of the ribbon. This result, in agreement with other recent calculations [141], seems to be in striking contrast with the simulations in Ref. [36]. More detailed analysis focusing on specific disorder models could unravel this ambiguity. Another very important issue, which might lead to different results, is the inclusion of hopping between second nearest neighbors in the tight-binding Hamiltonian. This would entail a coupling between carbon atoms belonging to the same sublattice and thus a current along the edges of the zGNR [147].

Weak Anderson disorder on the edges leads to the opening of a gap in the conductance of zigzag ribbons [143]. Some results are reported in Fig. 18. A weak potential on the edges has a deep impact on the electronic spectrum and then on the transport properties around the CNP. The states corresponding to the two almost flat bands at $E=0$ at the borders of the first Brillouin zone $(k= \pm \pi / a)$ are completely 


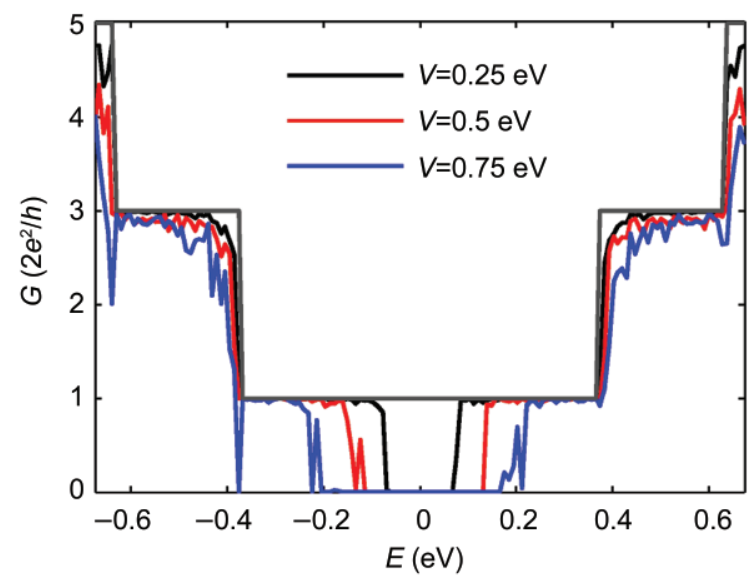

Figure 18 Differential conductance of a zigzag ribbon ( $N=32)$ with Anderson disorder on the edges. The energy of the edge sites varies randomly in the range $\pm V$ with $V=0.25,0.5$, and $0.75 \mathrm{eV}$ over a length of about $1 \mu \mathrm{m}$

localized on the edges of the ribbon. Therefore, a potential on the edges moves (upward or downward) the energy of these states. As a consequence, the electrons are subject to backscattering, especially in the region where most of the onsite disorder energies are concentrated, i.e., around the CNP. This induces a gap in the conductance, whose width increases with the strength of the potential.

We conclude this section by giving a look to the possible effects of edge roughness on the behavior of graphene-based (field-effect or Schottky-barrier fieldeffect) transistors [144-146, 148, 149]. These systems are usually investigated with the help of Schrödinger -Poisson solvers, which allow the self-consistent evaluation of the electrostatic potential. The results presented in the literature agree that edge disorder reduces the $I_{\text {on }}$ current and increases the $I_{\text {off }}$ current, thus making their ratio worse. The decreasing of $I_{\text {on }}$ is due to quantum transport effects, as seen before, and to self-consistent electrostatic effects that compensate the vacancies with an accumulation of charge on the atoms close to it. The increasing of the leakage current is due to the tunneling of electrons through the states induced in the band gap region or through conducting zigzag fragments. The degrading of the $I_{\text {on }} / I_{\text {off }}$ ratio is thus a serious issue for the efficiency of transistors based on very small ribbons. On the other hand, larger ribbons would reduce the gap tremendously. A recent theoretical analysis by Ouyang et al. [149] showed, in agreement with experiment [137], that the presence of optical phonons (OPs) considerably reduces the effect of edge irregularities. In fact, electrons lose a large amount of energy by emitting an OP and they can hardly return to the source after being reflected by the edges. As a consequence, elastic backscattering is only relevant when occurring close to the source, where electrons retain their energy before phonon emission.

\subsection{Minimum conductivity and charge mobility in graphene-based systems}

In semiconducting materials (such as silicon nanowires [121]), charge mobility is a very important quantity to assess the transport efficiency of the system and the corresponding device performance. By definition, the charge mobility is

$$
\mu(E)=\sigma_{\mathrm{sc}}(E) / e n(E)
$$

where

$$
\sigma_{\mathrm{sc}}=e^{2} \rho(E) v(E) l_{\mathrm{el}}
$$

is the semi-classical conductivity deduced from the Einstein formula, with $\rho(E)$ the DoS, $n(E)$ the charge density at energy $E, l_{\mathrm{el}}$ the elastic mean free path, and the elementary charge. Close to the charge neutrality (or Dirac) point, the measured experimental conductivity of various samples was found to be in the range $\sim 2-5 e^{2} / h$, although the charge mobility was changing by almost one order of magnitude [5-14]. This effect has been attributed to the change of charge density due to the doping from the substrate and/or contacts.

On the theoretical side, the calculation of the Kubo conductivity for 2-D graphene with short range disorder, and within the self-consistent Born approximation, yields $\sigma_{x x}^{\min }=4 e^{2} /(\pi h)(h$ is the Planck constant) for the two Dirac nodes [150], which is typically $1 / \pi$ smaller than all the experimental data. Numerical calculations using the Kubo formula confirm such a prediction [151, 153]. Amazingly, as discussed in Section 1.5.1, this value also comes out in a completely ballistic transport regime, as a contact effect.

In contrast, by assuming that the Dirac fermion scattering is dominated by Coulomb scattering from ionized impurities near the graphene plane, Nomura and MacDonald [151] could numerically reproduce

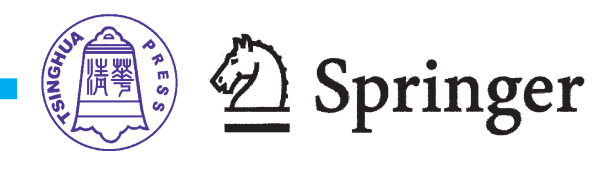


the low-energy dependence of the measured charge conductivity, and found that $\sigma_{x x}^{\min } \approx e^{2} / h$ close to the Dirac point, in better agreement with most experiments. In Ref. [151], the authors used the full quantum approach of the Kubo formula, describing the long range disorder effects via a screened Coulomb potential, and performing a finite size scaling analysis.

Other calculations within the semi-classical Boltzmann approach or the Landauer approach have also reported the effect of screened Coulomb potentials on charge conductivity [82, 154, 155] with similar conclusions, and although the existence of a true universal minimum conductivity could not be rigorously answered, an interpretation in terms of saturation of the conductivity due to charged impurity induced inhomogeneities, occurring at low densities, was proposed [154].

These results suggest that the intrinsic disorder in graphene could be of electrostatic (Coulomb scattering) nature, likely due to charges trapped in the oxide. We also note that the effect of graphene plane deformation modes (known as ripples), frozen when the exfoliated layer is deposited onto an oxide layer, has been investigated by introducing an effective random gauge potential $[97,156,157]$. The authors found that a temperature independent minimal conductivity will take place, with full suppression of localization effects owing to the absence of intervalley scattering processes, in agreement with temperature dependent experiments [21].

Concerning the contribution of quantum interference effects and the transition to an Andersontype localized regime, the issue is still controversial. In presence of strong intervalley scattering processes, which is best realized for short range disorder potential, conventional weak localization phenomena have been predicted [95, 158] and observed [123]. The preservation of the pseudospin symmetry also allows for the manifestation of a spectacular symmetry dependent anti-localization effect [95, 123, 158]. The transition to a localized Andersontype regime is more debated [99, 100], with no experimental evidence to date in such materials. The question is how quantum interference effects and localization phenomena for Dirac fermions depend on the underlying disorder potential characteristics, and whether the conventional 2-D scaling theory of localization $[104,105]$ is applicable or not in today's graphene materials.

Several authors have challenged the single parameter scaling theory of Anderson localization by studying the so-called beta function $\beta(g)=d \ln g / d \ln L$, with $g$ the dimensionless conductance and the system size. By computing the scaling behavior of $\beta(g)$, the localization versus delocalization nature of electronic states can be analyzed. In conventional 2-D systems, the theory predicts that all states are localized, independently of the disorder characteristics, provided time reversal symmetry is preserved [104, 105].

Recent numerical studies claim that in the presence of short range disorder (Anderson-type potential) and intervalley scattering, all states are indeed localized even for Dirac fermions [152]. However, it is interesting to note the typical values obtained for transport length scales. By varying the disorder strength from $W \approx 3.4 \mathrm{eV}$ to $W \approx 2 \mathrm{eV}$, the elastic mean free path was found to range from a few $\mathrm{nm}$ to several tens of nm close to the Dirac point (Fig. 19(a) inset), whereas the localization length $\xi$ given by $\xi=l_{\mathrm{e}} \exp \left(\pi \sigma_{\mathrm{sc}} / G_{0}\right)$ increased from $20 \mathrm{~nm}$ to $10 \mu \mathrm{m}$ (not shown here, see Ref. [152]). The energy dependence of $\xi$ was also shown to be strongly driven by that of the semi-classical conductivity with a minimum localization length at the Dirac point [152].

In case of long range disorder, the situation is more complex. Indeed, in the absence of intervalley scattering, Dirac fermions cannot be trapped by a potential well, irrespective of the well depth. This suggests the robustness of states against an insulating tendency. A different kind of scaling behavior of the conductance at the Dirac point was first proposed in Ref. [159]. The key result was the occurrence of a quantum critical point at half filling giving a universal value of the conductivity of the order of $e^{2} / h$, in contradiction with a localized nature which gives a zero conductivity in the thermodynamic limit. Other numerical studies [101, 160] found a different scaling flow for the beta function of the Dirac model, thus indicating that all states remain delocalized whatever the strength of the underlying disorder, but with a conductivity increasing with length up 


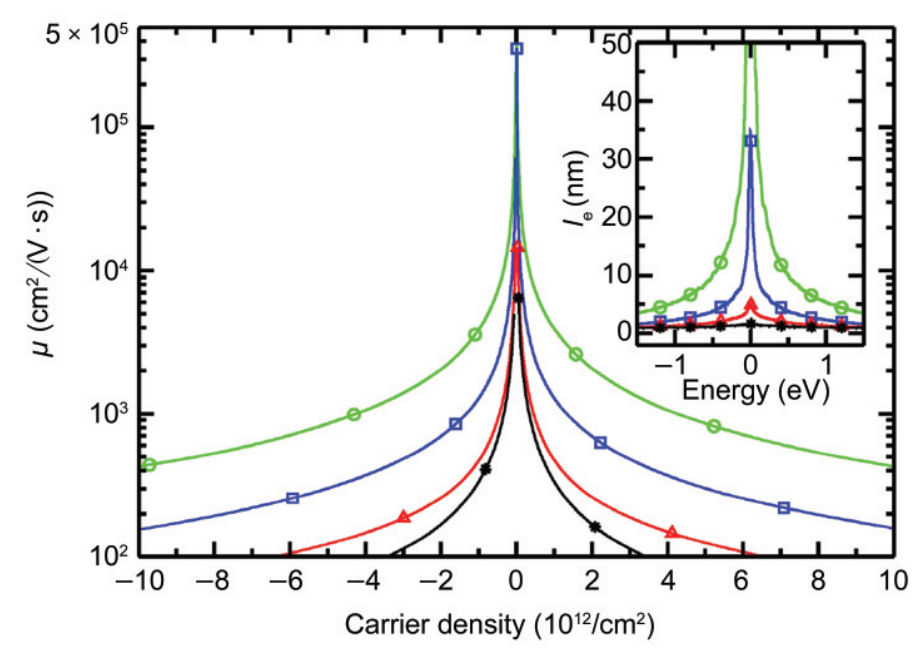

(a)

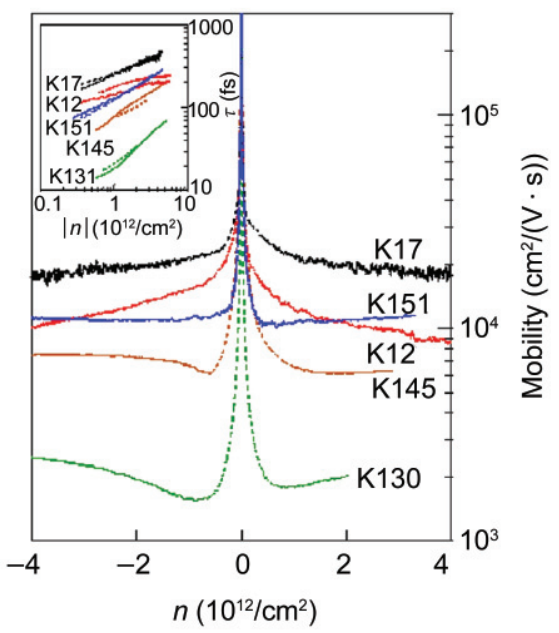

(b)

Figure 19 (a) Charge mobility as a function of carrier density and mean free path (inset) for $W=1,1.5,2,2.5$ (from top to bottom), from Ref. [152]. (b) Experimental mobility, from Ref. [165]

to infinity. The situation changes in the presence of ripples, which can be described by random vector potentials. Nomura and co-workers [156] further deepened the scaling behavior of both transport coefficients $\sigma_{x x}$ and $\sigma_{x y}$ and found that massless Dirac fermions exhibit a critical behavior similar to that of the quantum Hall transition point, but in the absence of uniform magnetic field.

An important observation is that to date, the theoretical description of disorder in graphene layers (either deposited on a substrate or suspended between contacts) has been mostly achieved at a phenomenological level. Usually, short range and long range scattering potentials are described by some onsite potential fluctuations (Anderson-type) and by Gaussian correlated potentials, respectively. Although the study of deformation modes known as ripples is at the origin of predictions of peculiar transport phenomena [101, 161], a realistic description of Coulomb scatterers is however needed to allow a true experimental exploration of localization phenomena in massless Dirac fermions. Novikov [162] first discussed the possible asymmetry in the transport cross section for a Dirac electron scattered by a positively or negatively charged Coulomb impurity. Self consistent RPA-Boltzmann theory also found Coulomb scattering induced conductivity asymmetry [163], without the possibility however to tackle this with localization effects. A recent theoretical study investigated quantum coherent transport and transport scaling lengths for intentionally chemically doped (and disordered) 2-D graphene layers with a realistic and self consistent description of impurity scattering potentials [164]. By incorporating substitutional boron (or nitrogen) impurities, elastic mean free paths, as well as the semi-classical conductivity and charge mobilities were numerically estimated by the Kubo approach. Some onset of quantum interference effects was also observed, even at the Dirac point, but this contribution was found to be too small to explore the possible underlying scaling behavior of the beta function, even in the case of strong doping (such as $4 \%$ ).

Finally, although conductivity is a well defined quantity, 2-D graphene manifests specific properties that make the use of Eq. (53) somehow ill-defined. Indeed, when the energy of charge carriers approaches the Dirac point, the semi-classical conductivity remains finite, whereas $\mu \rightarrow \infty$ as the charge density $n \rightarrow 0$.

In Fig. 19, we show the result of a numerical calculation using the Kubo approach (see Ref. [152] for details). The evolution of $\mu$ is shown as a function of the Anderson disorder strength $W$. The energy-dependence of $\mu(E)$ and $l_{\mathrm{el}}(E)$ are found to be similar. In the close vicinity of the Dirac point, the downscaling of $\mu$ with $W$ follows the Fermi Golden Rule prediction, while it diverges when approaching

\section{圈国 Springer}


the Dirac point. Experimental data from Ref. [165] are also shown in Fig. 19 (b) for comparison. Different samples with varying quality show a similar trend, although patterns from different samples can substantially differ in shape indicating fluctuations in the disorder characteristics.

It is clear that by further reducing the dimensionality of the graphene material, charge mobilities will be reduced. Recent transport measurements on nanoribbon-based field-effect transistors show mobilities in order of $\mu \approx 100-300 \mathrm{~cm}^{2} /(\mathrm{V} \cdot \mathrm{s})$ [137], which are thus reduced in comparison with the 2-D graphene measurements (reported values can be as large as $100.00 \mathrm{~cm}^{2} /(\mathrm{V} \cdot \mathrm{s})$ [7-14]). However, the lateral size reduction allows for a larger energy gap, which ensures more efficient field-effect efficiency.

\section{Conclusions}

To conclude, in this review we have reported on the basics of electronic and transport properties in low dimensional carbon-based materials including 2-D graphene, graphene nanoribbons and carbon nanotubes. It has been shown that although nanotubes and nanoribbons share similar electronic confinement properties due to their nanoscale lateral sizes, the effects of boundary conditions in the perpendicular direction with respect to the system axis trigger very different transport features when disorder is included. Close to the charge neutrality point, the robustness of armchair nanotubes against disorder is absent in armchair nanoribbons, which cannot be classified in the family of 1-D massless fermions, owing to edge-induced gap openings. Nanoribbons with zigzag symmetries are even more spectacularly sensitive to disorder owing to the edge state-driven lower transport dimensionality. In contrast, for charge carrier energies lying in the higher energy subbands, the properties of nanotubes and ribbons present similar features, with strong energy dependence of elastic mean free paths and localization phenomena.

Additionally, the transition from a quasi-1-D to a true 2-D system results in strong damping of disorder effects, with enhanced elastic mean free paths together with strong damping of quantum interferences. In particular, the study with Anderson disorder demonstrates that even in the strongest case of short range scattering potential (with possible short range potential fluctuations as large as $1 \mathrm{eV}$ ), the computed 2-D localization lengths remain in the range of several hundred nanometers to microns. One can thus conclude that to observe weak and strong localization regimes, the presence of edges as well as a reduced lateral size are essential factors.

Finally, the possibility to produce and control defect densities either through intentional doping or by irradiation techniques (that produce vacancy-type defects) could open spectacular avenues to explore quantum transport phenomena (including quantum Hall effects [166]) in low dimensional materials, for which a realistic description of both underlying electronic structures as well as superimposed disorder potentials would be theoretically possible, allowing unprecedented exploration of experimental data at a quantitative level.

\section{Acknowledgments}

We acknowledge fruitful and enlightening discussions with Tsuneya Ando, Hongjie Dai, Toshiaki Enoki, Philip Kim, Kentaro Nomura, Rudolf A. Roemer, Riichiro Saito, Miriam del Valle, and Carter T. White. This work was partially supported by the ANR/PNANO project ACCENT, by the FP7/ ICT/FET GRAND project, by the "Graphene Project" of CARNOT Institute-Leti, by the European Union project "Carbon Nanotube Devices at the Quantum Limit" (CARDEQ) under contract No. IST-021285, by the Volkswagen Stiftung under Grant No. I/78 340, by the DFG Priority Program "Quantum Transport at the Molecular Scale" SPP1243 and by DAAD. Computing time provided by the $\mathrm{ZIH}$ at the Dresden University of Technology is also acknowledged.

\section{References}

[1] Saito, R.; Dresselhaus, G.; Dresselhaus, M. S. Physical Properties of Carbon Nanotubes; Imperial College Press: London, 1998.

[2] Loiseau, A.; Launois, P.; Petit, P., Roche, S.; Salvetat, J. -P. Understanding Carbon Nanotubes from Basics 
to Applications; Lecture Notes in Physics, Vol. 677; Springer-Verlag: Berlin, Heidelberg, 2006.

[3] Jorio, A.; Dresselhaus, G.; Dresselhaus, M. S. Carbon Nanotubes: Advanced Topics in the Synthesis, Structure, Properties and Applications; Springer-Verlag: Berlin, Heidelberg, 2007.

[4] Charlier, J. C.; Blase, X.; Roche, S. Electronic and transport properties of nanotubes. Rev. Mod. Phys. 2007, 79, 677-732.

[5] Novoselov, K. S.; Geim, A. K.; Morozov, S. V.; Jiang, D.; Zhang, Y.; Dubonos, S. V.; Grigorieva, I. V.; Filrsov, A. A. Electric field effect in atomically thin carbon films. Science 2004, 306, 666-669.

[6] Berger, C.; Song, Z. M.; Li, X. B.; Wu, X. S.; Brown, N.; Naud, C.; Mayou, D.; Li, T. B.; Hass, J.; Marchenkov, A. N.; Conrad, E. H.; First, P. N.; de Heer, W. A. Electronic confinement and coherence in patterned epitaxial graphene. Science 2006, 312, 1191-1196.

[7] Zhang, Y. B.; Tan, Y. W.; Stormer, H. L.; Kim, P. Experimental observation of the quantum Hall effect and Berry's phase in graphene. Nature 2005, 438, 201-204.

[8] Zhang, Y.; Jiang, Z.; Small, J. P.; Purewal, M. S.; Tan, Y. -W.; Fazlollahi, M.; Chudow, J. D.; Jaszczak, J. A.; Stormer, H. L.; Kim, P. Landau-level splitting in graphene in high magnetic fields. Phys. Rev. Lett. 2006, 96, 136806.

[9] Geim, A. K.; Novoselov, K. S. The rise of graphene. Nat. Mater. 2007, 6, 183-191.

[10]Öezyilmaz, B.; Jarillo-Herrero, P.; Efetov, D.; Kim, P. Electronic transport in locally gated graphene nanoconstrictions. Appl. Phys. Lett. 2007, 91, 192107.

[11] Öezyilmaz, B.; Jarillo-Herrero, P.; Efetov, D.; Abanin, D. A.; Levitov, L. S.; Kim, P. Electronic transport and quantum Hall effect in bipolar graphene $\mathrm{p}-\mathrm{n}-\mathrm{p}$ junctions. Phys. Rev. Lett. 2007, 99, 166804.

[12] Jiang, Z; Zhang, Y; Stormer, H. L.; Kim, P. Quantum Hall states near the charge-neutral Dirac point in graphene. Phys. Rev. Lett. 2007, 99, 106802.

[13] Novoselov, K. S.; Jiang, Z.; Zhang, Y.; Morozov, S. V.; Stormer, H. L.; Zeitler, U.; Maan, J. C.; Boebinger, G. S.; Kim, P.; Geim, A. K. Room-temperature quantum Hall effect in graphene. Science 2007, 315, 1379.

[14] Wu, X.; Li, X.; Song, Z.; Berger, C.; de Heer, W. A. Weak antilocalization in epitaxial graphene: Evidence for chiral electrons. Phys. Rev. Lett. 2007, 98, 136801.

[15] Castro Neto, A. H; Guinea, F.; Peres, N. M. R.; Novoselov,
K. S.; Geim, A. K. The electronic properties of graphene. arXiv:0709.1163v2.

[16] Dayen, J. -F.; Mahmood, A.; Golubev, D. S.; RochJeune, I.; Salles, P.; Dujardin, E. Side-gated transport in FIB-fabricated multilayered graphene nanoribbons. arXiv:0712.2314v2, 2008.

[17] Lemme, M. C.; Echtermeyer, T. J.; Baus, M.; Kurz, H. A graphene field-effect device. IEEE Elect. Dev. L. 2007, 28, 282-284.

[18] Han, M. Y.; Öezyilmaz, B.; Zhang, Y.; Kim, P. Energy band-gap engineering of graphene nanoribbons. Phys. Rev. Lett. 2007, 98, 206805.

[19] Chen, Z.; Lin, Y. -M.; Rooks, M. J.; Avouris, P. Graphene nano-ribbon electronics. Physica E 2007, 40, 228-232.

[20] Echtermeyer, T. J.; Lemme, M. C.; Bolten, J.; Baus, M.; Ramsteiner, M.; Kurz, H. Graphene field-effect devices. Eur. Phys. J. Special Topics 2007, 148, 19-26.

[21] Morozov, S. V.; Novoselov, K. S.; Katsnelson, M. I.; Schedin, F.; Elias, D. C.; Jaszczak, J. A.; Geim, A. K. Giant intrinsic carrier mobilities in graphene and its bilayer Phys. Rev. Lett. 2008, 100, 016602.

[22] Javey, A.; Guo, J.; Wang, Q.; Lundstrom, M.; Dai, H. Ballistic carbon nanotube field-effect transistors. Nature 2003, 424, 654-657.

[23] Javey, A.; Guo, J.; Farmer, D. B.; Wang, Q.; Wang, D.; Gordon, R. G.; Lundstrom, M.; Dai, H. Carbon nanotube field-effect transistors with integrated ohmic contacts and high- $\kappa$ gate dielectrics. Nano Lett. 2004, 4, 447450 .

[24] Li, X. L.; Wang, X. R.; Zhang, L.; Lee, S.; Dai, H. J. Chemically derived, ultrasmooth graphene nanoribbon semiconductors. Science 2008, 319, 1229-1232.

[25] Anderson, P. W. Absence of diffusion in certain random lattices. Phys. Rev. 1958, 109, 1492-1505.

[26] McClure, J. W. Diamagnetism of graphite. Phys. Rev. 1956, 104, 666-671.

[27] Nakada, K.; Fujita, M.; Dresselhaus, G.; Dresselhaus, M. $\mathrm{S}$. Edge state in graphene ribbons: Nanometer size effect and edge shape dependence. Phys. Rev. B 1996, 54, 17954.

[28] Wakabayashi, K.; Fujita, M; Ajiki, H.; Sigrist, M. Electronic and magnetic properties of nanographite ribbons. Phys. Rev. B 1999, 59, 8271-8282.

[29] Wakabayashi, K. Theory of ballistic electron emission microscopy. Phys. Rev. B 2001, 64, 125408.

[30] Ezawa, M. Peculiar width dependence of the electronic

\section{凰商: Springer}


properties of carbon nanoribbons. Phys. Rev. B 2006, 73, 045432.

[31]Peres, N. M. R; Castro Neto, A. H.; Guinea, F. Conductance quantization in mesoscopic graphene. Phys. Rev. B 2006, 73, 195411.

[32] Brey, L.; Fertig, H. A. Electronic states of graphene nanoribbons studied with the Dirac equation. Phys. Rev. B 2006, 73, 235411.

[33] Muñoz-Rojas F.; Jacob, D.; Fernádez-Rossier, J.; Palacios, J. J. Coherent transport in graphene nanoconstrictions. Phys. Rev. B 2006, 74, 195417.

[34] Wang, Z. F.; Li, Q.; Zheng, H.; Ren, H.; Su, H.; Shi, Q. $W_{\text {.; }}$ Chen, J. Tuning the electronic structure of graphene nanoribbons through chemical edge modification: A theoretical study. Phys. Rev. B 2007, 75, 113406.

[35] Zheng, H.; Wang, Z. F.; T. Luo, T.; Shi, Q. W.; Chen, J. Analytical study of electronic structure in armchair graphene nanoribbons. Phys. Rev. B 2007, 75, 165414.

[36] Areshkin, D. A.; Gunlycke, D.; White, C. T. Ballistic transport in graphene nanostrips in the presence of disorder: Importance of edge effects. Nano Lett. 2007, 7, 204-210.

[37]Gunlycke, D.; Areshkin, D. A.; White, C. T. Semiconducting graphene nanostrips with edge disorder. Appl. Phys. Lett. 2007, 90, 142104.

[38]Gunlycke, D.; Lawler, H. M.; White, C. T. Roomtemperature ballistic transport in narrow graphene strips. Phys. Rev. B 2007, 75, 085418.

[39] Fujita, M.; Wakabayashi, K.; Nakada, K.; Kusakabe, K. Peculiar localized state at zigzag graphite edge. J. Phys. Soc. Jpn. 1996, 65, 1920-1923.

[40] Fabrizio, M.; Parola, A.; Tosatti, E. Strong-coupling phases of two Hubbard chains with interchain hopping. Phys. Rev. B 1992, 46, 3159-3162.

[41] Gopalan, S.; Rice, T. M.; Sigrist, M. Spin ladders with spin gaps: A description of a class of cuprates. Phys. Rev. $B$ 1994, 49, 8901-8910.

[42] Choi, H. J.; Ihm, J.; Louie, S. G.; Cohen, M. L. Defects, quasibound states, and quantum conductance in metallic carbon nanotubes. Phys. Rev. Lett. 2000, 84, 29172920.

[43] Kim, G.; Lee, S. B.; Kim, T. -S.; Ihm, J. Fano resonance and orbital filtering in multiply connected carbon nanotubes. Phys. Rev. B 2005, 71, 205415.

[44] Kim, G.; Jeong, B. W.; Ihm, J. Deep levels in the band gap of the carbon nanotube with vacancy-related defects
Appl. Phys. Lett. 2006, 88, 193107.

[45] Zhou, T.; Wu, J.; Duan, W.; Gu, B. -L. Physical mechanism of transport blocking in metallic zigzag carbon nanotubes. Phys. Rev. B 2007, 75, 205410.

[46] Sánchez-Portal, D.; Ordejón, P.; Artacho, E.; Soler, J. M. Density-functional method for very large systems with LCAO basis sets. Int. J. Quantum Chem. 1997, 65, 453461.

[47] Malysheva, L.; Onipko, A. Spectrum of $\pi$ electrons in graphene as a macromolecule. Phys. Rev. Lett. 2008, 100, 186806.

[48] Cresti, A.; Grosso G.; Pastori Parravicini, G. Electronic states and magnetotransport in unipolar and bipolar graphene ribbons. Phys. Rev. B 2008, 77, 115408.

[49] Sutton, A.P. Electronic Structure of Materials; Clarendon Press: Oxford, 1994, p. 41.

[50] Rycerz, A.; Tworzydło, J.; Beenaker, C. W. J. Valley filter and valley valve in graphene. Nat. Phys. 2007, 3, 172175.

[51] Akhmerov, A. R.; Bardarson, J. H.; Rycerz, A.; Beenaker, C. W. J. Theory of the valley-valve effect in graphene nanoribbons. Phys. Rev. B 2008, 77, 205416.

[52] Li, Z.; Qian, H.; Wu, J.; Gu, B. -L.; Duan, W. Role of symmetry in the transport properties of graphene nanoribbons under bias. Phys. Rev. Lett. 2008, 100, 206802.

[53] Cresti, A.; Grosso, G.; Pastori Parravicini, G. Valleyvalve effect and even-odd chain parity in $\mathrm{p}-\mathrm{n}$ graphene junctions. Phys. Rev. B 2008, 77, 233402.

[54] Cresti, A; Grosso, G.; Pastori Parravicini, G. Fieldeffect resistance of gated graphitic polymeric ribbons: Numerical simulations. Phys. Rev. B 2008, 78, 115433.

[55] Miyamoto, Y.; Nakada, K.; Fujita, M. First-principles study of edge states of $\mathrm{H}$-terminated graphitic ribbons. Phys. Rev. B 1999, 59, 9858-9861.

[56] Kawai, T.; Miyamoto, Y.; Sugino, O.; Koga, Y. Graphitic ribbons without hydrogen-termination: Electronic structures and stabilities. Phys. Rev. B 2000, 62, R16349.

[57] Son, Y. -W.; Cohen, M. L.; Louie, S. G. Half-metallic graphene nanoribbons. Nature 2006, 444, 347-349.

[58] Barone, V.; Hod, O.; Scuseria, G. E. Electronic structure and stability of semiconducting graphene nanoribbons Nano Lett. 2006, 6, 2748-2754.

[59] Son, Y. -W.; Cohen, M. L.; Louie, S. G. Energy gaps in graphene nanoribbons. Phys. Rev. Lett. 2006, 97, 216803. 
[60] White, C. T.; Li, J.; Gunlycke, D.; Mintmire, J. W. Hidden one-electron interactions in carbon nanotubes revealed in graphene nanostrips. Nano Lett. 2007, 7, 825-830.

[61] Fujita, M.; Igami, M.; Nakada, K. Lattice distortion in nanographite ribbons. J. Soc. Phys. Jpn. 1997, 66, 18641867.

[62] Sasaki, K.; Murakami, S.; Saito, R. Stabilization mechanism of edge states in graphene. Appl. Phys. Lett. 2006, 88, 113110.

[63] Gunlycke, D.; White, C. T. Tight-binding energy dispersions of armchair-edge graphene nanostrips. Phys. Rev. B 2008, 77, 115116.

[64] Klusek, Z.; Waqar, Z.; Denisov, E. A.; Kompaniets, T. N.; Makarenko, I. W.; Titkov, A. N.; Bhatti, A. S. Observations of local electron states on the edges of the circular pits on hydrogen-etched graphite surface by scanning tunneling spectroscopy. Appl. Surf. Sci. 2000, $161,508-514$.

[65] Niimi, Y.; Matsui, T.; Kambara, H.; Tagami, K.; Tsukada, M.; Fukuyama, H. Scanning tunneling microscopy and spectroscopy studies of graphite edges. Appl. Surf. Sci. 2005, 241, 43-48.

[66] Kobayashi, Y.; Fukui, K.; Enoki, T.; Kusakabe, K.; Kaburagi, Y. Observation of zigzag and armchair edges of graphite using scanning tunneling microscopy and spectroscopy. Phys. Rev. B 2005, 71, 193406.

[67] Wallace, P. R. The band theory of graphite. Phys. Rev. 1947, 71, 622-634.

[68] Reich, S.; Maultzsch, J.; Thomsen, C.; Ordejón P. Tightbinding description of graphene. Phys. Rev. B 2002, 66, 035412.

[69] Grüneis, A.; Attaccalite, C.; Wirtz, L.; Shiozawa, H.; Saito, R.; Pichler, T.; Rubio, A. Tight-binding description of the quasiparticle dispersion of graphite and few-layer graphene. arXiv:0808.1467v2, 2008.

[70] Fiori, G.; Iannaccone, G. Simulation of graphene nanoribbon field-effect transistors. IEEE Elect. Dev. $L$. 2007, 28, 760-762.

[71] Sun, L.; Li, Q.; Ren, H.; Shi, Q. W.; Yang, J.; Hou, J. G. Strain effect on energy gaps of armchair graphene nanoribbons. arXiv:cond-mat/0703795, 2007.

[72] Gunlycke, D.; Areshkin, D. A.; Li, J.; Mintmire, J. W.; White, C. T. Graphene nanostrip digital memory device. Nano Lett. 2007, 7, 3608-3611.

[73] Palacios, J. J.; Fernández-Rossier, J.; Brey, L. Vacancyinduced magnetism in graphene and graphene ribbons.
Phys. Rev. B 2008, 77, 195428.

[74] Fernández-Rossier, J. Prediction of hidden multiferroic order in graphene zigzag ribbons. Phys. Rev. B 2008, 77, 075430.

[75] Nemec, N.; Tománek, D.; Cuniberti, G. Contact dependence of carrier injection in carbon nanotubes: An ab initio study. Phys. Rev. Lett. 2006, 96, 076802.

[76] Nemec, N.; Tománek, D.; Cuniberti, G. Modeling extended contacts for nanotube and graphene devices. Phys. Rev. B 2008, 77, 125420.

[77] Tworzydło, J.; Trauzettel, B.; Titov, M.; Rycerz, A.; Beenakker, C. W. J. Sub-Poissonian shot noise in graphene. Phys. Rev. Lett. 2006, 96, 246802.

[78] Kastnelson, M. I. Zitterbewegung, chirality, and minimal conductivity in graphene. Eur. Phys. J. B 2006, 51, 157160.

[79] Cresti, A.; Grosso, G.; Pastori Parravicini, G. Numerical study of electronic transport in gated graphene ribbons. Phys. Rev. B 2007, 76, 205433.

[80] Schomerus, H. Effective contact model for transport through weakly-doped graphene. Phys. Rev. B 2007, 76, 045433.

[81] San-Jose, P.; Prada, E.; Golubev, D. S. Universal scaling of current fluctuations in disordered graphene. Phys. Rev. $B$ 2007, 76, 195445.

[82] Lewenkopf, C. H.; Mucciolo, E. R.; Castro Neto, A. H. Numerical studies of conductivity and Fano factor in disordered graphene. Phys. Rev. B 2008, 77, 081410.

[83] Miao, F.; Wijeratne, S.; Zhang, Y.; Coskun, U. C.; Bao, W.; Lau, C. N. Phase-coherent transport in graphene quantum billiards. Science 2007, 317, 1530-1533.

[84] DiCarlo, L.; Williams, J. R.; Zhang, Y.; McClure, D. T.; Marcus, C. M. Shot noise in graphene. Phys. Rev. Lett. 2008, 100, 156801.

[85] Danneau, R.; Wu, F.; Craciun, M. F.; Russo, S.; Tomi, M. Y.; Salmilehto, J.; Morpurgo A. F.; Hakonen, P. J. Shot noise in ballistic graphene. Phys. Rev. Lett. 2008, 100, 196802.

[86] Nemec, N; Cuniberti, G. Hofstadter butterflies of bilayer graphene. Phys. Rev. B 2007, 75, 201404.

[87] Nemec, N; Cuniberti, G. Hofstadter butterflies of carbon nanotubes: Pseudofractality of the magnetoelectronic spectrum. Phys. Rev. B 2006, 74, 165411.

[88] Brey, L.; Fertig, H. A. Electronic states of graphene nanoribbons studied with the Dirac equation. Phys. Rev. B 2006, 73, 235411.

[89] Novoselov, K. S.; Geim, A. K.; Morozov, S. V.; Jiang, D.; 
Katsnelson, M. I.; Grigorieva, I. V.; Dubonos, S. V.; Firsov, A. A. Two-dimensional gas of massless Dirac fermions in graphene. Nature 2005, 438, 197-200.

[90] Gusynin, V. P.; Sharapov, S. G. Unconventional integer quantum Hall effect in graphene. Phys. Rev. Lett. 2005, 95, 146801.

[91]Cresti, A. Proposal for a graphene-based current nanoswitch. Nanotechnology 2008, 19, 265401.

[92] Moser, J.; Barreiro, A.; Bachtold, A. Current-induced cleaning of graphene. App. Phys. Lett. 2007, 91, 163513.

[93] Ando, T.; Nakanishi, T.; Saito, R. Berry's phase and absence of back scattering in carbon nanotubes. J. Phys. Soc. Jpn. 1998, 67, 2857-2862.

[94] White, C. T.; Todorov, T. N. Carbon nanotubes as long ballistic conductors. Nature 1998, 393, 240-242.

[95] McCann, E.; Kechedzhi, K.; Fal'ko, V. I.; Suzuura, H.; Ando, T.; Altshuler, B. L. Weak-localization magnetoresistance and valley symmetry in graphene. Phys. Rev. Lett. 2006, 97, 146805.

[96] Ostrovsky, P. M.; Gornyi, I. V.; Mirlin, A. D. Electron transport in disordered graphene. Phys. Rev. B 2006, 74, 235443.

[97] Morpurgo A. F.; Guinea, F. Intervalley scattering, longrange disorder, and effective time-reversal symmetry breaking in graphene. Phys. Rev. Lett. 2006, 97, 196804.

[98] Khveshchenko, D. V. Effects of long-range correlated disorder on Dirac fermions in graphene. Phys. Rev. $B$ 2007, 75, 241406.

[99] Suzuura, H.; Ando, T. Weak-localization in metallic carbon nanotubes. J. Phys. Soc. Jpn. 2006, 75, 024703.

[100] Aleiner, I. L.; Efetov, K. B. Effect of disorder on transport in graphene. Phys. Rev. Lett. 2006, 97, 236801.

[101]Nomura, K.; Koshino, M.; Ryu, S. Topological delocalization of two-dimensional massless Dirac fermions. Phys. Rev. Lett. 2007, 99, 146806.

[102] Anderson, P. W. Localized magnetic states in metals. Phys. Rev. 1961, 124, 41-52.

[103] Abrahams, E.; Anderson, P. W.; Licciardello, D. C.; Ramakrishnan T. V. Scaling theory of localization: absence of quantum diffusion in two dimensions. Phys. Rev. Lett. 1979, 42, 673-676

[104] Lee, P. A.; Fisher, D. S. Anderson localization in two dimensions. Phys. Rev. Lett. 1981, 47, 882-885.

[105] Lee, P. A.; Ramakrishnan, T. V. Disordered electronic systems. Rev. Mod. Phys. 1985, 57, 287-337.
[106] Roche, S.; Saito, R. Magnetoresistance of carbon nanotubes: From molecular to mesoscopic fingerprints. Phys. Rev. Lett. 2001, 87, 246803.

[107] Fedorov, G.; Lassagne, B.; Sagnes, M.; Raquet, B.; Broto, J. -M.; Triozon, F.; Roche, S.; Flahaut, E. Gatedependent magnetoresistance phenomena in carbon nanotubes. Phys. Rev. Lett. 2005, 94, 066801.

[108] Gomez-Navarro, C.; Pablo, P. J. De; Gómez-Herrero, J.; Biel, B.; Garcia-Vidal, F. J.; Rubio, A.; Flores, F. Tuning the conductance of single-walled carbon nanotubes by ion irradiation in the Anderson localization regime. Nat. Mater. 2005, 4, 534-539.

[109] Biel, B.; Garcia-Vidal, F. J.; Rubio, A.; Flores, F. Anderson localization in carbon nanotubes: Defect density and temperature effects. Phys. Rev. Lett. 2005, 95, 266801.

[110] Strunk, C.; Stojetz, B.; Roche, S. Quantum interference in multiwall carbon nanotubes. Semicond. Sci. Technol. 2006, 21, S38-\$45.

[111] Fedorov, G.; Tselev, A.; Jimenez, D.; Latil, S.; Kalugin, N.; Barbara, P.; Roche, S. Magnetically induced field effect in carbon nanotube devices. Nano Lett. 2007, 7, 960964.

[112] Lassagne, B.; Cleuziou, J. -P., Nanot, S.; Escofier, W.; Avriller, R.; Roche, S.; Forro, L.; Raquet, B.; Broto, J. -M. Aharonov-Bohm conductance modulation in ballistic carbon nanotubes. Phys. Rev. Lett. 2007, 98, 176802.

[113] Stojetz, B.; Roche, S.; Miko, C.; Triozon, F.; Forro, L.; Strunk, C. Competition between magnetic field dependent band structure and coherent backscattering in multiwall carbon nanotubes. New J. Phys. 2007, 9, 56.

[114] Biel, B.; Garcia-Vidal, F. J.; Rubio, A.; Flores, F. Ab initio study of transport properties in defected carbon nanotubes: $\mathrm{An} \mathrm{O}(\mathrm{N})$ approach. J. Phys.: Condens. Matter 2008, 20, 294214.

[115] Flores, F.; Biel, B.; Rubio, A.; Garcia-Vidal, F. J.; GomezNavarro, C.; de Pablo, P. J.; Gomez-Herrero, J. Anderson localization regime in carbon nanotubes: Size dependent properties. J. Phys.: Condens. Matter 2008, 20, 304211.

[116] Hügle, S.; Egger, R. van Hove singularities in disordered multichannel quantum wires and nanotubes. Phys. Rev. B 2002, 66, 193311.

[117] Nemec, N., Richter, K.; Cuniberti, G. Diffusion and localization in carbon nanotubes and graphene nanoribbons. New J. Phys. 2008, 10, 065014. 
[118] Abrikosov, A.; Gor'kov, L. P.; Dzyaloshiuskii, I. E. Quantum Field Theoretical Methods in Statistical Physics; Pergamon: Oxford, 1965.

[119] López Sancho, M. P.; López Sancho, J. M.; Rubio, J. Highly convergent schemes for the calculation of bulk and surface Green functions. J. Phys. F: Met. Phys. 1985, 15, 851-858.

[120] Nemec, N. Quantum transport in carbon-based nanostructures. PhD Thesis, Universität Regensburg, 2007.

[121] Lherbier, A.; Persson, M.; Niquet, Y. M.; Triozon, F.; Roche, S. Quantum transport length scales in siliconbased semiconducting nanowires: Surface roughness effects. Phys. Rev. B. 2008, 77, 085301.

[122] Triozon, F.; Roche, S.; Rubio, A.; Mayou, D. Electrical transport in carbon nanotubes: Role of disorder and helical symmetries. Phys. Rev. B 2004, 69, 121410.

[123] Tikhonenko, F. V.; Horsell, D. W.; Gorbachev, R. V.; Savchenko, A. K. Weak localization in graphene flakes. Phys. Rev. Lett. 2008, 100, 056802.

[124] Morozov, S. V.; Novoselov, K. S.; Katsnelson, M. I.; Schedin, F.; Ponomarenko, L. A.; Jiang, D.; Geim, A. K. Strong suppression of weak localization in graphene. Phys. Rev. Lett. 2006, 97, 016801.

[125] Datta, S. Electronic Transport in Mesoscopic Systems; Cambridge University Press: New York, NY, 1995.

[126] Thouless, D. J. Maximum metallic resistance in thin wires. Phys. Rev. Lett. 1977, 39, 1167-1169.

[127] Beenakker, C. W. J. Random-matrix theory of quantum transport. Rev. Mod. Phys. 1997, 69, 731-808.

[128] Avriller, R.; Latil, S.; Triozon, F.; Blase, X.; Roche, S. Chemical disorder strength in carbon nanotubes: xMagnetic tuning of quantum transport regimes. Phys. Rev. B 2006, 74, 121406.

[129] Niimi, Y.; Matsui, T.; Kambara, H.; Tagami, K.; Tsukada, M.; Fukuyama, H. Scanning tunneling microscopy and spectroscopy of the electronic local density of states of graphite surfaces near monoatomic step edges. Phys. Rev. B. 2006, 73, 085421.

[130] Enoki, T.; Kobayashi, Y.; Katsuyama, C.; Osipov, V. Y.; Baidakova, M. V.; Takai, K.; Fukui, K.; Vul', A. Y. Structures and electronic properties of surface/edges of nanodiamond and nanographite. Diamond Relat. Mater. 2007, 16, 2029-2034.

[131] Kobayashi, Y.; Fukui, K.; Enoki, T.; Kusakabe, K. Edge state on hydrogen-terminated graphite edges investigated by scanning tunneling microscopy. Phys. Rev. B 2006, 73, 125415.

[132] Enoki, T.; Kobayashi, Y.; Fukui, K. I. Electronic structures of graphene edges and nanographene. Int. Rev. Phys. Chem. 2007, 26, 609-645.

[133] Banerjee, S.; Sardar, M.; Gayathri, N.; Tyagi, A. K.; Raj, B. Conductivity landscape of highly oriented pyrolytic graphite surfaces containing ribbons and edges. Phys. Rev. B 2005, 72, 075418.

[134] Banerjee, S.; Sardar, M.; Gayathri, N.; Tyagi, A. K.; Raj, B. Enhanced conductivity in graphene layers and at their edges. Appl. Phys. Lett. 2006, 88, 602111.

[135] Cançado, L. G.; Pimenta, M. A.; Neves, B. R.; Dantas, M. S.; Jorio, A. Influence of the atomic structure on the Raman spectra of graphite edges. Phys. Rev. Lett. 2004, 93, 247401.

[136] de Heer, W. A.; Berger, C.; Wu, X.; First, P. N.; Conrad, E. H.; Li, X.; Li, T.; Sprinkle, M.; Hass, J.; Sadowski, M. L.; Potemski, M.; Martinez, G. Epitaxial graphene. Solid State Commun. 2007, 143, 92-100.

[137] Wang, X.; Ouyang, Y.; Li, X.; Wang, H.; Guo, J.; Dai, $\mathrm{H}$. Room-temperature all-semiconducting sub-10-nm graphene nanoribbon field-effect transistors. Phys. Rev. Lett. 2008, 100, 206803.

[138] Tapasztó, L.; Dobrik, G.; Lambin, P.; Biró, L. P. Tailoring the atomic structure of graphene nanoribbons by scanning tunnelling microscope lithography. Nature Nanotech. 2008, 3, 397-401.

[139] Querlioz, D.; Apertet, Y.; Valentin, A.; Huet, K.; Bournel, A.; Galdin-Retailleau, S.; Dollfus, P. Suppression of the orientation effects on bandgap in graphene nanoribbons in the presence of edge disorder. Appl. Phys. Lett. 2008, 92, 042108.

[140] Evaldsson, M.; Zozoulenko, I. V.; Xu, H.; Heinzel, T. Edge disorder induced Anderson localization and conduction gap in graphene nanoribbons. arXiv:0805.4326v1, 2008.

[141] Mucciolo, E. R.; Castro Neto, A. H.; Lewenkopf, C. $\mathrm{H}$. Conductance quantization and transport gap in disordered graphene nanoribbons. arXiv:0806.3777, 2008.

[142] Martin, I.; Blanter, Y. M. Transport in disordered graphene nanoribbons. arXiv:0705.0532v2, 2008.

[143] Li, T. C.; Lu, S. -P. Quantum conductance of graphene nanoribbons with edge defects. Phys. Rev. B. 2008, 77, 085408. 
[144] Yoon, Y.; Guo, J. Effect of edge roughness in graphene nanoribbon transistors. Appl. Phys. Lett. 2007, 91, 073103.

[145] Fiori, G.; Yoon, Y.; Hong, S.; lannaccone, G.; Guo, J. Performance comparison of graphene nanoribbon Schottky barrier and MOS FETs. In Int. Electron Device Meeting (IEDM) Tech. Dig. 2007, pp. 757-760.

[146] Basu, D.; Gilbert, M. J.; Register, L. F.; Banerjee, S. K.; MacDonald, A. H. Effect of edge roughness on electronic transport in graphene nanoribbon channel metal-oxide-semiconductor field-effect transistors. Appl. Phys. Lett. 2008, 92, 042114.

[147] Wimmer, M.; Adagideli, I.; Berber, S.; Tománek, D; Richter, K. Spin transport in rough graphene nanoribbons. Phys. Rev. Lett. 2008, 100, 177207.

[148] Yoon, Y.; Fiori, G.; Hong, S.; lannaccone, G.; Guo, J. Performance comparison of graphene nanoribbon FETs with Schottky contacts and doped reservoirs. IEEE Transactions on Electron Devices 2008, 55, 23142323.

[149] Ouyang, Y.; Wang, X.; Dai, H.; Guo, J. Carrier scattering in graphene nanoribbon transistors. Appl. Phys. Lett. 2008, 92, 243124.

[150] Shon N. H.; Ando, T. Quantum transport in twodimensional graphite system. J. Phys. Soc. Jpn. 1998, 67, 2421-2429.

[151] Nomura K.; MacDonald, A. H. Quantum Hall ferromagnetism in graphene. Phys. Rev. Lett. 2006, 96, 256602.

[152] Lherbier, A.; Biel, B.; Niquet, Y. M.; Roche, S. Transport length scales in disordered graphene-based materials: Strong localization regimes and dimensionality effects. Phys. Rev. Lett. 2008, 100, 036803.

[153] Peres, N. M. R.; Guinea, F.; Castro Neto, A. H. Electronic properties of disordered two-dimensional carbon. Phys. Rev. B 2008, 73, 125411.

[154] Hwang, E. H.; Adam, S.; Das Sarma, S. Carrier transport in two-dimensional graphene layers Phys. Rev. Lett. 2007, 98, 186806.
[155] Dong, H. M.; Xu, W.; Zeng, Z.; Peeters, F. M. Quantum and transport conductivities in monolayer graphene. Phys. Rev. B 2008, 77, 235402.

[156] Nomura, K.; Ryu S.; Koshino, M.; Mudry, C.; Furusaki A. Quantum Hall effect of massless Dirac fermions in a vanishing magnetic field. Phys. Rev. Lett. 2008, 100, 246806.

[157] Kim, E. -A.; Castro Neto, A. H. Graphene as an electronic membrane. arXiv:cond-mat/0702562v2, 2008.

[158] Suzuura, H.; Ando, T. Crossover from symplectic to orthogonal class in a two-dimensional honeycomb lattice. Phys. Rev. Lett. 2002, 89, 266603.

[159] Ostrovsky, P. M.; Gornyi, I. V.; Mirlin, A. D. Quantum criticality and minimal conductivity in graphene with long-range disorder. Phys. Rev. Lett. 2007, 98, 256801.

[160] Bardarson, J. H.; Tworzydło, J.; Brouwer, P. W.; Beenakker, C. W. J. One-parameter scaling at the Dirac point in graphene. Phys. Rev. Lett. 2007, 99, 106801.

[161] Guinea, F.; Katsnelson, M. I.; Vozmediano, M. A. $\mathrm{H}$. Midgap states and charge inhomogeneities in corrugated graphene. Phys. Rev. B 2008, 77, 075422.

[162] Novikov, D. S. Numbers of donors and acceptors from transport measurements in graphene. Appl. Phys. Lett. 2007, 91, 102102.

[163] Adam, S.; Hwang, E. H.; Galitski, V. M.; Das Sarma, S. A self-consistent theory for graphene transport. PNAS. 2007, 104, 18392.

[164] Lherbier, A.; Blase X., M.; Niquet, Y. M.; Triozon, F.; Roche $S$. Charge transport in chemically doped 2D graphene. Phys. Rev. Lett. 2008, 101, 036808.

[165] Tan, Y. W.; Zhang, Y.; Bolotin, K.; Zhao, Y.; Adam, S.; Hwang, E. H.; Das Sarma, S.; Stormer, H. L.; Kim, $P$. Measurement of scattering rate and minimum conductivity in graphene. Phys. Rev. Lett. 2007, 99, 246803.

[166] Koshino, M.; Ando, T. Hall plateau diagram for the Hofstadter butterfly energy spectrum. Phys. Rev. B 2006, 73, 155304. 